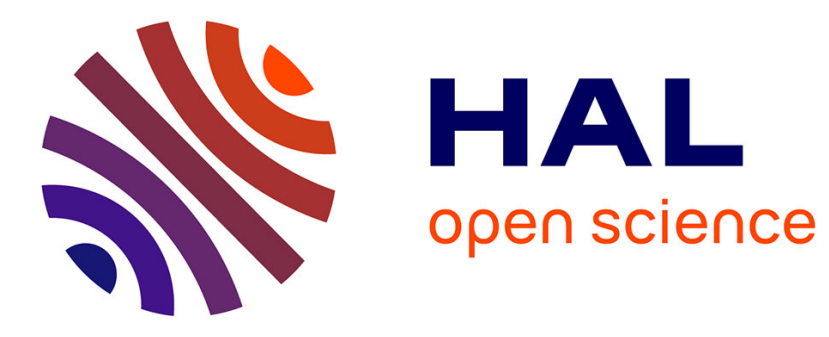

\title{
Effect of carbon insertion on the structural and magnetic properties of NdScSi
}

Tadhg Mahon, Etienne Gaudin, Antoine Villesuzanne, Bernard Chevalier, Sophie Tencé

\section{- To cite this version:}

Tadhg Mahon, Etienne Gaudin, Antoine Villesuzanne, Bernard Chevalier, Sophie Tencé. Effect of carbon insertion on the structural and magnetic properties of NdScSi. Inorganic Chemistry, 2019, 58 (22), pp.15255-15268. 10.1021/acs.inorgchem.9b02260 . hal-02376882

\section{HAL Id: hal-02376882 \\ https://hal.science/hal-02376882}

Submitted on 8 Sep 2020

HAL is a multi-disciplinary open access archive for the deposit and dissemination of scientific research documents, whether they are published or not. The documents may come from teaching and research institutions in France or abroad, or from public or private research centers.
L'archive ouverte pluridisciplinaire HAL, est destinée au dépôt et à la diffusion de documents scientifiques de niveau recherche, publiés ou non, émanant des établissements d'enseignement et de recherche français ou étrangers, des laboratoires publics ou privés. 


\title{
The effect of carbon insertion on the structural and magnetic properties of $\mathrm{NdScSi}$
}

\section{Authors}

Tadhg MAHON, Etienne GAUDIN , Antoine VILLESUZANNE, Bernard CHEVALIER and Sophie TENCÉ*

CNRS, Univ. Bordeaux, Bordeaux INP, ICMCB, UMR 5026, F-33600 Pessac, France

\section{*sophie.tence@icmcb.cnrs.fr}

\begin{abstract}
The investigation of layered intermetallic compounds containing light elements like hydrogen has great potential for superconductivity. We studied the insertion of carbon atoms in $\mathrm{CeScSi}$ type intermetallics (an ordered variant of the $\mathrm{La}_{2} \mathrm{Sb}$ structure type) and here we report the new carbide $\mathrm{NdScSiC}_{0.5}$. Carbon insertion keeps the pristine compound's space group, $14 / \mathrm{mmm}$, but causes an anisotropic expansion of the unit cell with an increase in the $a$ parameter and a decrease of the $c$ parameter. $X$-ray and neutron diffraction measurements indicate the existence of a $\mathrm{NdScSiC}_{x}$ solid solution $(0.2<\mathrm{x} \leq 0.5)$ with carbon atoms occupying only the $\mathrm{Sc}_{4} \mathrm{Nd}_{2}$ octahedral sites while leaving the $\mathrm{Nd}_{4}$ tetrahedral sites vacant. Magnetization measurements unveil a linear reduction of the ferromagnetic ordering temperature from $T_{C}$ $\approx 171 \mathrm{~K}$ to $\approx 50 \mathrm{~K}$ with increasing carbon content. The ferromagnetic structures of the pristine $\mathrm{NdScSi}$ and the filled $\mathrm{NdScSiC}_{0.5}$ have been determined from neutron diffraction measurements. Finally, we discuss the effect of carbon versus hydrogen insertion on electronic and magnetic properties based on density functional theory calculations. Although the unpaired spin density channels between $\mathrm{Nd}$ and Sc atoms (responsible of the high Curie temperature in $\mathrm{NdScSi}$ ) are reduced upon carbon insertion, the strong $\mathrm{Nd}-\mathrm{C}$ interaction, linked to a reduced $c$ lattice parameter in $\mathrm{NdScSiC}_{0.5}$, ensures a strong magnetic coupling between $\mathrm{Nd}$ double layer along the $c$-axis and the ferromagnetic order is preserved.
\end{abstract}

KEYWORDS

Carbide; CeScSi-type intermetallic; magnetic properties; magnetic structures; DFT calculations

\section{Introduction}

The insertion of hydrogen in intermetallics has been well investigated both for the possibility of modulating the physical properties of the parent compound and for hydrogen storage. In particular, the hydrogenation of equiatomic $R T X(R=$ rare-earth, $T=$ transition metal, $X=\mathrm{p}$ block element) intermetallics has attracted much attention ( ${ }^{1}$ and references herein). For example, the hydrogenation of the CeFeSi-type compound LaFeSi induces non-conventional iron-based superconductivity in the hydride LaFeSiH ${ }^{2}$. Most $R T X$ intermetallics are able to 
accept hydrogen atoms into their vacant interstitial sites, in particular, those adopting the $\mathrm{CeScSi}$-structure type ${ }^{3-9}$ (a ordered variant of the $\mathrm{La}_{2} \mathrm{Sb}$ structure type ${ }^{10}$ ). Hydrogenation of these materials produces an anisotropic expansion of the unit cell with the $a$ parameter decreasing and the $c$ parameter strongly increasing (e.g. in NdScSi $a=4.2894(6) \AA$ decreases to $4.221(1) \AA$ while $c=15.705$ (3) $\AA$ increases to $16.928(2) \AA{ }^{7}$ ). In our recent work on the hydrogenation of $\mathrm{NdScSi}$ we demonstrated by in-situ neutron diffraction study that octahedral/square-based pyramidal $\left(\mathrm{Sc}_{4} \mathrm{Nd}_{2} / \mathrm{Sc}_{4} \mathrm{Nd}\right)$ sites were also available for hydrogen insertion along with the previously known tetrahedral sites ${ }^{7}$. An identical structure was found for the hydride $\mathrm{CeTiGeH}_{1.5}$ obtained from both the high-temperature form (CeScSi-type) and the the low-temperature form (CeFeSi-type) of CeTiGe ${ }^{8}$. This points out that two types of interstitial sites can be occupied by $\mathrm{H}$ atoms in the CeScSi-structure type. Note also that Mizoguchi et al. reported the same structure for the hydride $\mathrm{LaTiAsH}_{1.15}$ synthesized directly by high pressure ${ }^{11}$. Hydrogen insertion in CeScSi-type intermetallics usually leads to a drastic reduction of the magnetic ordering temperature of the parent compounds. The Curie temperature $T_{C}$ of $\mathrm{NdScSi}$ is reduced from $171 \mathrm{~K}$ to around $4 \mathrm{~K}$ on hydrogen insertion and from $350 \mathrm{~K}$ to $6 \mathrm{~K}$ for GdScGe ${ }^{6,7}$. Similarly, while GdTiGe is ferromagnetic below $376 \mathrm{~K}$, the corresponding hydride, GdTiGeH, does not display magnetic order down to $4 \mathrm{~K}^{3}$. The case of Ce compounds is less systematic. Indeed, the hydrogenation of CeScGe and CeScSi yields a strong decrease of the antiferromagnetic ordering temperature from $T_{N}=46(26) \mathrm{K}$ to $3 \mathrm{~K}$ for $X=\mathrm{Ge}(\mathrm{Si})$ and the occurrence of a Kondo type behaviour ${ }^{5}$. In contrast, the insertion of hydrogen into the non-magnetic Kondo germanide CeTiGe leads to the appearance of a magnetic ordering at $3.5 \mathrm{~K}^{8}$. In this case, hydrogenation plays the role of a negative pressure, tuning the system from the non-magnetic to the Kondo magnetic part of the Doniach phase diagram.

The insertion of other light elements such as boron or carbon is understandably more difficult to achieve in RTX phases. Yet, the presence of a vacant octahedral site in the CeScSi-type materials raises the possibility of inserting these elements into these compounds. Indeed, carbon atoms typically occupy octahedral sites in compounds such as $\mathrm{Mn}_{5} \mathrm{Si}_{3}$-type phases ${ }^{12-}$ 14, $\mathrm{La}\left(\mathrm{Fe}_{1-\mathrm{x}} X_{\mathrm{x}}\right)_{13}$ compounds $(X=\mathrm{Si}, \mathrm{Al} \text { or } \mathrm{Ga})^{15,16}$ and $R_{2} \mathrm{Fe}_{17}$-type systems $(R=\mathrm{Ce}, \mathrm{Nd}, \mathrm{Sm}, \mathrm{Ho}$, $\mathrm{Er}$, etc. $)^{17-21}$. Carbon insertion was largely investigated in these intermetallics, especially, to improve the magnetic properties of those used as permanent magnets or magnetocalorics materials. Carbon atoms occupy $R_{6}$ octahedral sites in rare-earth based $\mathrm{Mn}_{5} \mathrm{Si}_{3}$-type phases or mixed $R_{2} \mathrm{Fe}_{4}$ cavities in $\mathrm{La}\left(\mathrm{Fe}_{1-\mathrm{x}} X_{\mathrm{x}}\right)_{13}$ compounds and $R_{2} \mathrm{Fe}_{17}$-type systems. In the Fe-rich phases, this generally results in an augmentation of the Curie temperature, linked to the increase of the Fe-Fe distances and thus exchange interactions. To date, however, no work has been published on the insertion of carbon into materials with the CeScSi-structure type and more generally into RTX intermetallics. Only two theses mention the possibility of inserting carbon and boron atoms in Ti-based $R T X$ compounds ${ }^{22,23}$. Nevertheless, in the light of our results, insertion of $\mathrm{C}$ or $\mathrm{B}$ in the Ti-based systems deserves further studies to confirm the preliminary theses' results. Indeed, there is not significant modification of the cell 
parameters and magnetic properties after insertion compared to the pristine CeScSi-type phases ${ }^{3,4,24}$. The main argument of the authors' theses to justify $B$ or $C$ insertion is the change of the structure-type from CeFeSi to $\mathrm{CeScSi}$ thanks to insertion. However, we have since demonstrated that one can stabilize the CeScSi-type structure only by high temperature annealing ${ }^{8,24}$. We present here, for the first time, a thorough study of the insertion of carbon into the CeScSi-type compound NdScSi. The effects of carbon insertion on the crystal and magnetic structures and magnetic properties are described. We also compare the electronic structures of the pristine $\mathrm{NdScSi}$, the hydride $\mathrm{NdScSiH} 1.5$ and the carbide $\mathrm{NdScSiC}_{0.5}$.

\section{Experimental}

Stoichiometric amounts of the metals ( $\mathrm{Sc}-99.999 \%$ supplied by Neyco, $\mathrm{Nd}-99.99 \%$, supplied by STREM chemicals, Si - 99.9999\% supplied by Alfa Aesar) were cut, polished and weighed before being cleaned in an arc furnace (Edmund Bühler GmbG MAM1 Compact Arc Melter with a $230 \mathrm{~V}$ connection) under argon atmosphere using a titanium getter to remove residual oxygen. The cleaned metals were then polished again to remove any residual oxide before being melted together with carbon, cut from a cold pressed graphite pellet, in an induction furnace. The resulting ingot was weighed before being melted a further three times in the arc furnace to ensure complete melting of any residual binary carbides, turning between each fusion. Total mass loss was generally less than $1 \%$. The resulting intermetallic buttons exhibit a metallic, silvery lustre with a faceted interior once cut and show no sign of oxidation/decomposition after several months in air. The target carbide phase is present in the as cast ingots. To improve crystallinity and homogeneity the ingots were wrapped in tantalum foil and annealed in evacuated quartz ampoules for 2 weeks at $900^{\circ} \mathrm{C}$. However, annealing at $900^{\circ} \mathrm{C}$ produces only a slight improvement in the crystallinity and haves little to no effect on the overall purity of the sample. A common secondary phase in all samples was

$\mathrm{Sc}_{2} \mathrm{CO}$ which crystallises in the rock salt structure with $a=4.5906 \AA$. This phase likely forms from a reaction between residual scandium oxide and the carbon during the initial synthesis. Additionally, some other silicides or carbides can be present in low concentration ( 3-4 wt\%) such as $\mathrm{NdSi}_{2-x}, \mathrm{NdSi}, \mathrm{Nd}_{5-x} \mathrm{Sc}_{x} \mathrm{Si}_{3}, \mathrm{Nd}_{2} \mathrm{ScSi}_{2}, \mathrm{Nd}_{5} \mathrm{Si}_{3} \mathrm{C}$ or $\mathrm{NdC}_{2}$. The relative amount of these secondary phases depends on the nominal composition and obviously increases when the carbon proportion exceeds the limits of carbon solubility in NdScSi.

Routine powder X-ray diffraction (PXRD) was performed with the use of a PANalytical X'pert Pro diffractometer ( $\mathrm{Cu}-\mathrm{K} \alpha$ radiation) for the structural characterisation and phase identification of the as-cast and annealed samples. X-ray powder data for Rietveld analysis were collected at room temperature using a PANalytical $X^{\prime}$ pert Pro diffractometer working with the $\mathrm{Cu}-\mathrm{K} \alpha_{1}(1.54051 \AA)$ radiation in the range $10 \leq 2 \theta \leq 130^{\circ}$ and a step size of $0.008^{\circ}$. The powders used were ground and sieved to have particle sizes of less than $45 \mu \mathrm{m}$. Rietveld refinement and full pattern matchings were carried out using the Fullprof program package ${ }^{25}$. 
Single crystal X-ray diffraction data were collected at room temperature on an Enraf-Nonius Kappa CCD using Mo K $\alpha$ radiation. The single crystal was selected on the basis of the size and sharpness of the diffraction spots. A Gaussian-type absorption correction was applied, the shape of the single crystal being determined with the video microscope of the diffractometer. 2034 reflections were measured up to $\theta=35^{\circ}$. Data processing and all refinements were performed with the Jana2006 program package. ${ }^{26}$ At the end of the refinement the reliability factors were $\mathrm{R}\left[\mathrm{F}^{2}>2 \sigma\left(\mathrm{F}^{2}\right)\right]=0.015$ and $\mathrm{wR}\left(\mathrm{F}^{2}\right)=0.044$. $\mathrm{R} / \mathrm{Rw}, \mathrm{S}=1.11$ for 14 parameters, 231 independent reflections and with residual electron density in the range $\left[-0.56,+0.60 \mathrm{e}^{-} \AA^{-3}\right]$. Details of data collections and structure refinement can be found in the crystallographic information file (CIF) deposited in the joint CCDC/FIZ Karlsruhe deposition service with reference CSD 1939969.

Magnetization measurements were performed using a superconducting quantum interference device (SQUID) magnetometer (Quantum Design MPMS-XL) in the temperature range $2-300 \mathrm{~K}$ and in fields up to $7 \mathrm{~T}$.

Neutron powder diffraction was carried out on $5 \mathrm{~g}$ (approximately $1 \mathrm{~cm}^{3}$ ) of $\mathrm{NdScsi}$ and $\mathrm{NdScSiC}_{0.5}$ at the $\mathrm{G} 4.1$ beamline at the LLB (Laboratoire Léon Brillouin) at Saclay, France. The wavelength was $2.428 \AA$ and measurements were made using vanadium sample holders in the temperature ranges $1.5-200 \mathrm{~K}$.

Total carbon content determination was carried out by combustion analysis using an Eltra CS800 carbon/sulphur determinator. The total carbon content is measured using CS-800 infrared cells to detect $\mathrm{CO}_{x}$ species and quantify them with respect to a known standard. The samples are placed in a disposable ceramic crucible and metallic accelerators are added to facilitate complete combustion. For our experiments $700 \mathrm{mg}$ of high purity iron filings and 1.5 $\mathrm{g}$ of tungsten (provided by Eltra $\mathrm{GmbH}$ ) were added as accelerants following the recommendation of the manufacturer. Approximately $50 \mathrm{mg}$ of sample were burned in pure oxygen with the accelerants in a disposable ceramic crucible by heating in a $2.2 \mathrm{~kW}$ induction furnace. To remain in the accurate detection zone, the mass of sample used was varied based on nominal carbon mass (lower masses for more carbon rich samples). Pure oxygen allows the consumption of all the carbon present to form $\mathrm{CO}_{\mathrm{x}}$ gases which pass through a dust filter to the detectors. To calibrate the instrument, the carbon content of a cast iron standard with known carbon content (3.39(2) ppm C, Eltra GmbH) was measured three times using the same accelerant proportions described above. Approximately $25 \mathrm{mg}$ of the standard was used for each calibration. The accelerants were then measured without the sample or cast iron standard to provide a baseline (again, three times) before the samples were measured. The total carbon content was then used in conjunction with phase quantification by Rietveld refinement to give an estimate of the effective carbon content of the main phase.

Density functional theory (DFT) calculations were performed using the projector augmented-wave (PAW) method as implemented in the VASP code. ${ }^{27-29}$ PAW datasets were used to describe valence electron-ionic core interactions, with valence $N d 4 f, 5 s, 5 p, 5 d, 6 s$, 
Sc $3 p, 3 d, 4 s$, Si 3s, 3p, C 2s, $2 p$ and $H$ 1s electrons treated explicitly. The correlation energy was treated at the generalized gradient approximation (GGA) level, according to the PerdewBurke-Ernzerhof formulation. ${ }^{30}$ The exchange energy was improved using the Heyd-ScuseriaErnzerhof (HSE06) screened hybrid exchange functional, where 25\% of GGA exchange is replaced by exact Hartree-Fock (HF) exchange. ${ }^{31,32} \Gamma$-centered Brillouin zone samplings of $8 \times 8 \times 2 k$-points were used.

Although their usage involves drastically larger computing times than pure DFT exchange functionals, DFT-Hartree-Fock hybrid functionals improve significantly the accuracy of calculated band gaps and crystal structures: optimized lattice parameters and atomic positions lie then typically within a percent of the experimental data. ${ }^{33}$ Therefore, we chose to use the crystal data from experiment as input, all along our calculations. In particular, we do not investigate here lattice dynamics, which would better require atomic positions and lattice parameters corresponding to calculated energy minima; little deviations of the structural parameters from the optimal calculated values will not affect significantly the density of states nor the charge and spin densities, of primary interest here.

\section{Results and discussion}

\section{X-ray diffraction}

\section{The $\mathrm{NdScSiC}_{x}(0.2<x \leq 0.5)$ solid solution}

From the $X$-ray diffraction measurements carbon insertion conserves the symmetry of the pristine material with an anisotropic expansion of the unit cell as shown in Figure 1 for $x=0.5$. The carbides, like the pristine parent compounds, crystallise in the $14 / \mathrm{mmm}$ space group and carbon insertion causes an increase in the $a$ parameter while decreasing the $c$ parameter. 


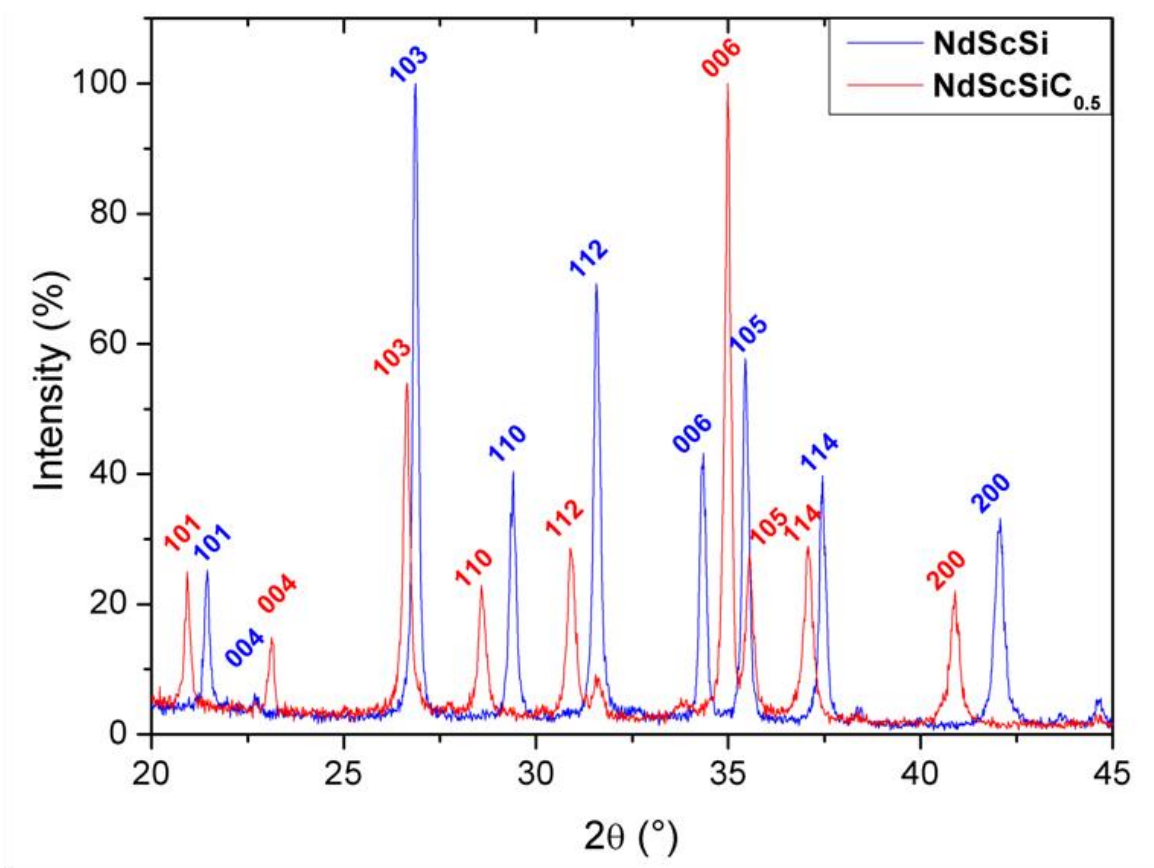

Figure 1. X-ray diffraction patterns of annealed $\mathrm{NdScSi}$ and $\mathrm{NdScSiC} \mathrm{C}_{0.5}$ with peaks indexed with the $14 / \mathrm{mmm}$ space group.

The X-ray diffraction patterns of $\mathrm{NdScSiC}_{x}$ showed that, for low nominal concentrations of carbon $(x \leq 0.2)$ the XRD patterns contain two sets of peaks: one of these can be easily assigned to the pristine $\mathrm{NdScSi}$ compound while the second corresponds to the new carbide. As the carbon content is increased, the change in the unit cell parameters of the carbide phase becomes more pronounced and the intensity of the peaks for the pristine phase decreases. After approximately $x=0.2$ the pristine phase is no longer observed in X-ray diffraction measurements. The unit cell parameters of the carbide phase continue the trend ( $a$ increasing and $c$ decreasing) until $x=0.5$ where they reach a plateau as shown in Figure 2 . Thus carbon insertion in NdScSi leads to an increase in the a parameter from $4.289 \AA$ to $4.412 \AA$ (+2.86\%) and a decrease of the $c$ parameter from $15.705 \AA$ to $15.382 \AA$ (-2.06 \%) in NdScSiC 0.5 (Table 1). Increasing $x$ above 0.5 quickly leads to the formation of binary rare earth and scandium carbides at the expense of the target $\mathrm{NdScSiC}_{0.5}$ phase.

Table 1. Unit cell parameters of $\mathrm{NdScSi}$ and $\mathrm{NdScSiC}_{0.5}$ refined from powder XRD data.

\begin{tabular}{llllll}
\hline & $\boldsymbol{a}(\AA)$ & $c(\AA)$ & $\mathbf{V}\left(\AA^{3}\right)$ & $c / a$ & reference \\
\hline NdScSi & $4.2894(6)$ & $15.705(3)$ & 288.96 & 3.661 & 7 \\
NdScSiC $_{0.5}$ & $4.412(2)$ & $15.382(6)$ & 299.38 & 3.486 & This work \\
$\Delta(\%)$ & +2.86 & -2.06 & +3.61 & -4.78 & \\
\hline
\end{tabular}




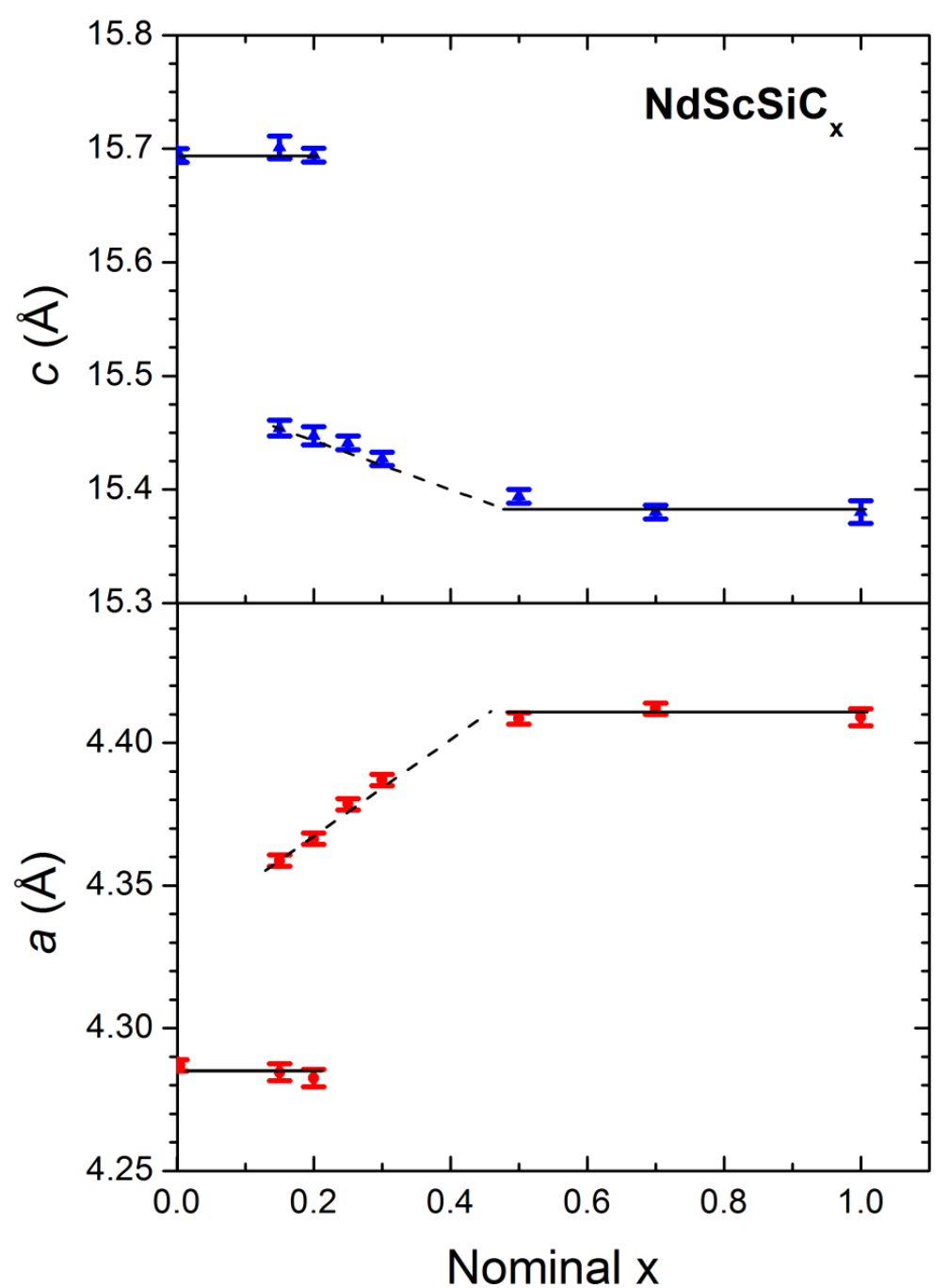

Figure 2. Unit cell parameters against nominal carbon content $x$ for the $\mathrm{NdScSiC}_{\mathrm{x}}$ system. Guide lines are added to help visualise trends.

Due to the presence of secondary carbides in all samples, the nominal $x$ value does not correspond to the actual carbon content of the $\mathrm{NdScSiC}_{\mathrm{x}}$ phases (e.g. see in Figure $\mathrm{S} 1$ the Rietveld refinement of the XRD pattern obtained for nominal $x=0.6$ ). Therefore the effective $x$ value was estimated by the following method: the total carbon content present in the entire sample was determinated through combustion analysis and then assigned to the different carbide phases according to their relative quantification given by Rietveld refinement. Due to the presence of non-stoichiometric secondary carbide phases in many samples, only three samples were selected for this treatment, i.e. samples containing only stoichiometric binary carbides as secondary phases and spaning the zone of solubility. The refined unit cell parameters are plotted against the calculated effective $x$ values in Figure 3 . As expected, the $a$ parameter increases with increasing carbon content while the $c$ parameter decreases. A linear relationship between the unit cell parameters and effective $\mathrm{x}$ is seen. If the fit line for the $\mathrm{Nd}$ system is extended beyond 0.25 (dashed line), it can be seen that it intersects the point for the pristine NdScSi. This linear relationship was used with Vegard's law to determine 
the effective $x$ value for the other $\mathrm{NdScSiC}_{\mathrm{x}}$ samples based on their unit cell parameters. From these data it can be concluded that, unlike the hydride systems described previously ${ }^{7}$, the insertion of carbon into the CeScSi-type intermetallics produces a solid solution. The $\mathrm{NdScSiC}_{x}$ system has a composition range of $0.2<x \leq 0.5$. This stoichiometry is lower than that observed for $\mathrm{NdScSiH}_{1.5}$, showing clearly that not all of the interstitial sites can be fully occupied by carbon.
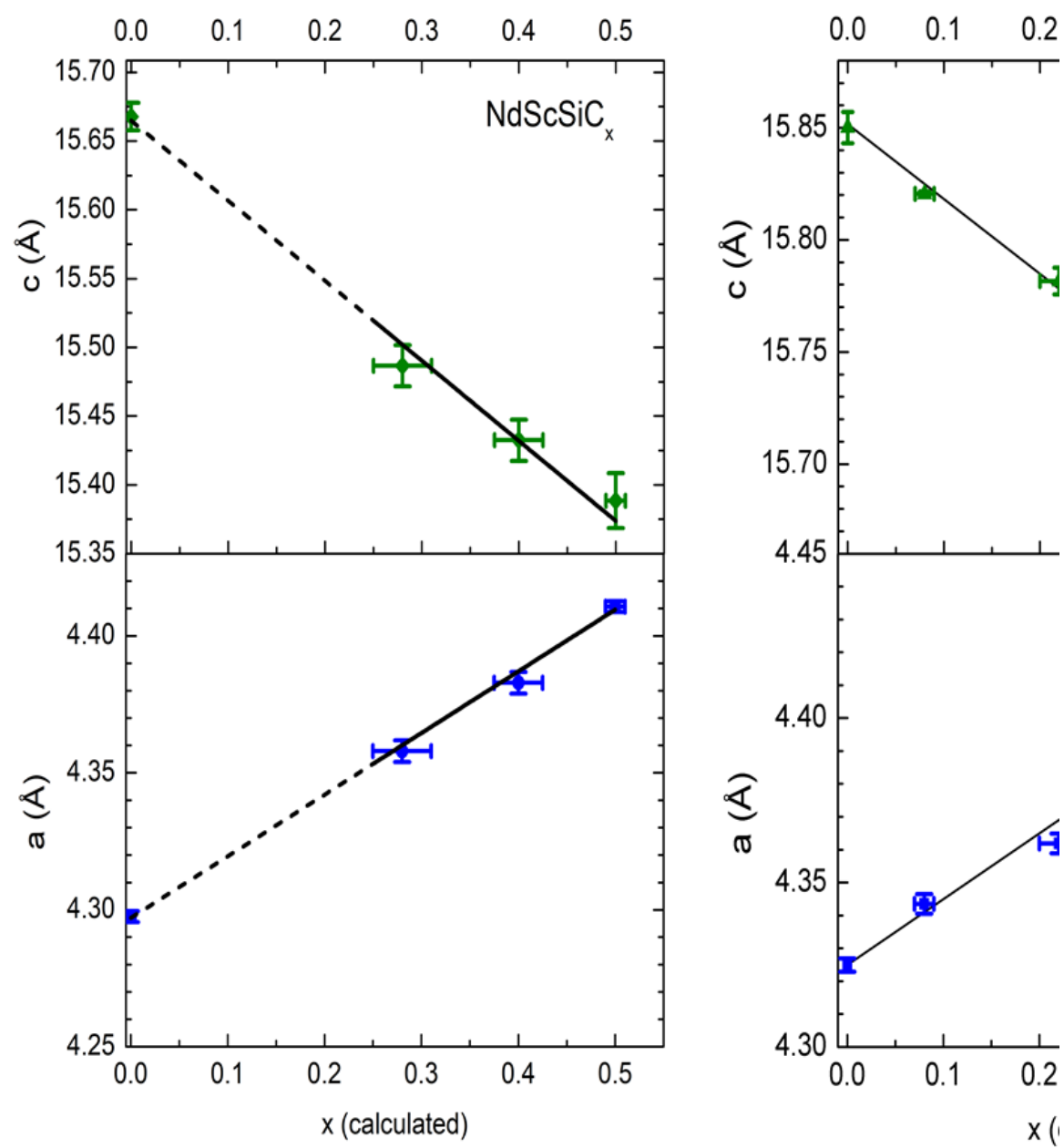

Figure 3. Unit cell parameters against calculated carbon content for the $\mathrm{NdScSiC}_{\mathrm{x}}$ system. $\mathbf{Y}$ error bars show $3 \sigma$ while $x$ error bars show $\sigma$. The full black lines show a linear fit of the data while the dotted black lines shows a linear extrapolation of the fit line through the biphasic zone.

\section{Single Crystal XRD on NdScSiC 0.5}

A single crystal of $\mathrm{NdScSiC}_{0.5}$ was isolated and measured using single crystal X-ray diffraction. The measurement confirms that the intermetallic carbide conserves the $\mathrm{CeScSi}$ structure type with $14 / \mathrm{mmm}$ space group. A Fourier difference map of the refinement without the $C$ atoms can be seen in Figure 4 (a). A significant residual electron density can clearly be observed at 
the centre of the Sc square planes before the inclusion of carbon. After the inclusion of carbon in this $2 b\left(\begin{array}{lll}0 & 0 \mathrm{1} / 2\end{array}\right)$ site there is no significant electron residual density observed anywhere in the cell (Figure 4 (b)). The refined atomic parameters for $\mathrm{NdScSiC}_{0.5}$ are given in Table 2 and the crystal structure of $\mathrm{NdScSi}$ and $\mathrm{NdScSiC}_{0.5}$ are shown in Figure 5 . The refinement confirms that the $2 b$ site is fully occupied by carbon atom and also reveals a small mixing of $\mathrm{Sc}$ and $\mathrm{Nd}$ atoms on the $\mathrm{Sc} 4 c$ site as that observed previously on $\mathrm{Gd}_{1.02} \mathrm{Sc}_{0.98} \mathrm{Ge}^{34}$, leading to the chemical formula $\mathrm{Nd}_{1.02} \mathrm{~S}_{0.98} \mathrm{SiC}_{0.5}$. With the ideal formula $\mathrm{NdScSiC}_{0.5}$ the equivalent atomic displacement parameter (ADP) of Sc position $\left(=0.0047 \AA^{2}\right)$ was significantly lower than the ADPs of the other positions (around $0.007 \AA^{2}$ ) and when the occupancy factor for $C$ position was released it converged to 1.31(6) instead of 1 . When the extra position $\mathrm{Nd} 2$ was introduced (Table 2), the equivalent ADP of Sc position was close the other ADPs and the occupancy factor for $C$ position converged to 1.11(7). The occupancy factor for $C$ was then fixed to the ideal value 1 and the full occupancy of this position is confirmed by its equivalent ADP value which is nearly equal to the other ones. Therefore, the carbon atoms are located only in the centre of the $\mathrm{Sc}_{4} \mathrm{Nd}_{2}$ octahedral sites, inside of the Sc square planes. This is contrary to the insertion of hydrogen detailed earlier ${ }^{7}$ where the hydrogen atoms occupy a site slightly above or below the Sc square plane as well as the empty Nd tetrahedra.
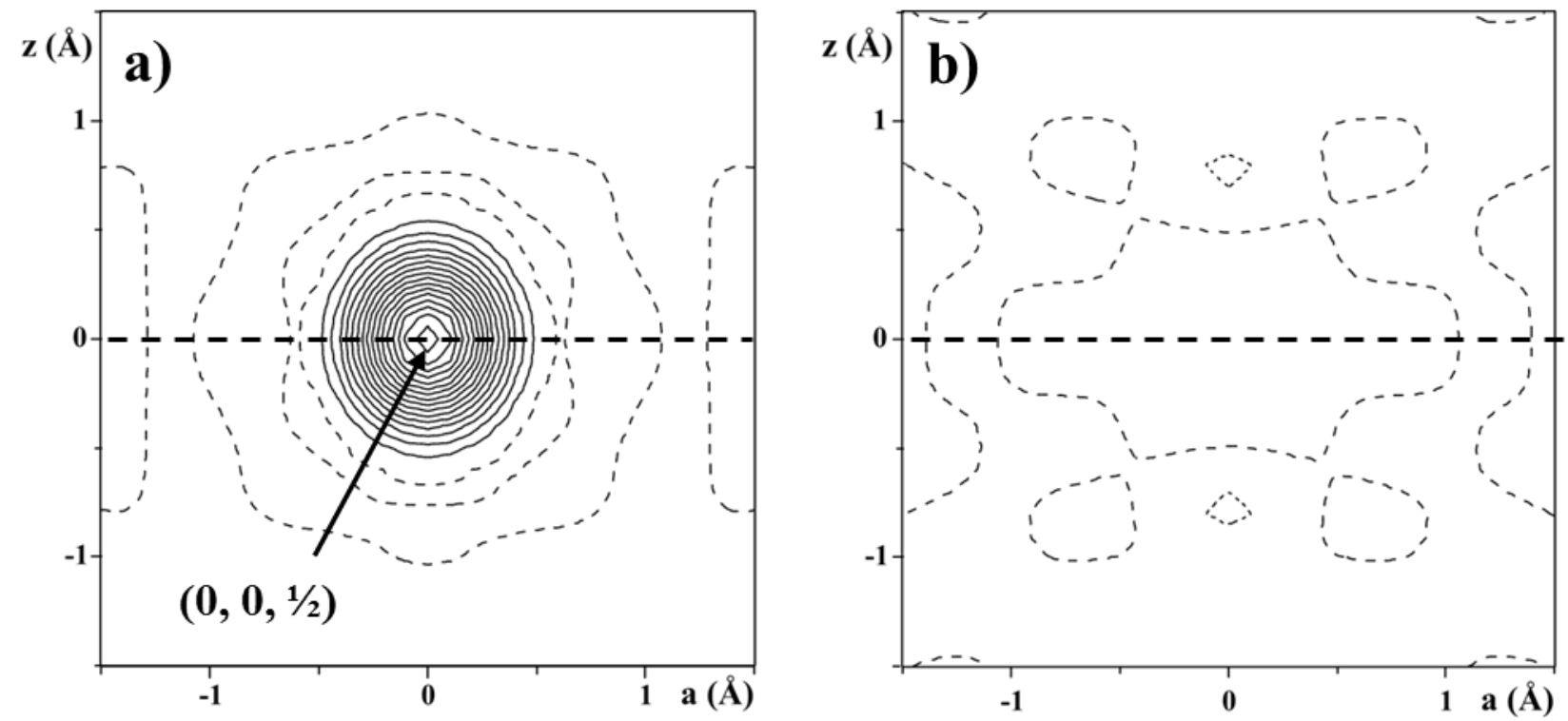

Figure 4. Difference Fourier maps for the single crystal refinement of $\mathrm{NdScSiC}_{0.5}$. (a) before the inclusion of $C$ in the $2 b$ Wyckoff site $\left(00 \frac{1}{2}\right)$, contour lines for 0 (dashed lines) and from 1 to $16 \mathrm{~A} \mathrm{e.} \AA^{-3}$ in intervals of 1 e. $\mathrm{A}^{-3}$ (continuous lines) (b) after the inclusion, contour lines for 0 and $-0.5 e . \AA^{-3}$ (dashed lines). 
Table 2. Atomic parameters refined from the single crystal XRD measurements on $\mathrm{Nd}_{1.02} \mathrm{Sc}_{0.98} \mathrm{SiC}_{0.5}\left(\mathrm{Nd}_{1.023(3)} \mathrm{Sc}_{0.977(3)} \mathrm{SiC}_{0.5}\right)$

\begin{tabular}{lllllll}
\hline $\begin{array}{l}\text { Atomic } \\
\text { positions }\end{array}$ & $\begin{array}{l}\text { Wyckoff } \\
\text { Positions }\end{array}$ & $\mathbf{x}$ & $\mathbf{y}$ & $\mathbf{z}$ & $\mathbf{U}_{\text {eq }}\left(\AA^{2}\right)$ & Occupancy \\
\hline $\mathbf{N d 1}$ & $4 e$ & 0 & 0 & $0.33308(2)$ & $0.00736(9)$ & 1 \\
$\mathbf{N d 2}$ & $4 c$ & 0 & $1 / 2$ & 0 & $0.0068(3)$ & $0.023(3)$ \\
$\mathrm{Sc1}$ & $4 c$ & 0 & $1 / 2$ & 0 & 0.0068 & $0.977(3)$ \\
$\mathrm{Si}$ & $4 e$ & 0 & 0 & $0.12853(10)$ & $0.0065(3)$ & 1 \\
$\mathbf{C}$ & $2 b$ & 0 & 0 & $1 / 2$ & $0.0068(12)$ & 1 \\
\hline Space group $14 / \mathrm{mmm}$ & $a=4.4157(5) \quad c=15.381(2) \AA$ \\
Refinement parameters $: \mathrm{R}\left(\mathrm{F}^{2}\right) / \mathrm{R}_{\text {wall }}\left(\mathrm{F}^{2}\right)=1.52 \% / 4.42 \% \quad \mathrm{~S}=1.10$ \\
$\Delta \rho_{\max }$ and $\Delta \rho_{\min }\left(\mathrm{e} . \AA^{-3}\right)=0.60$ and -0.56 &
\end{tabular}
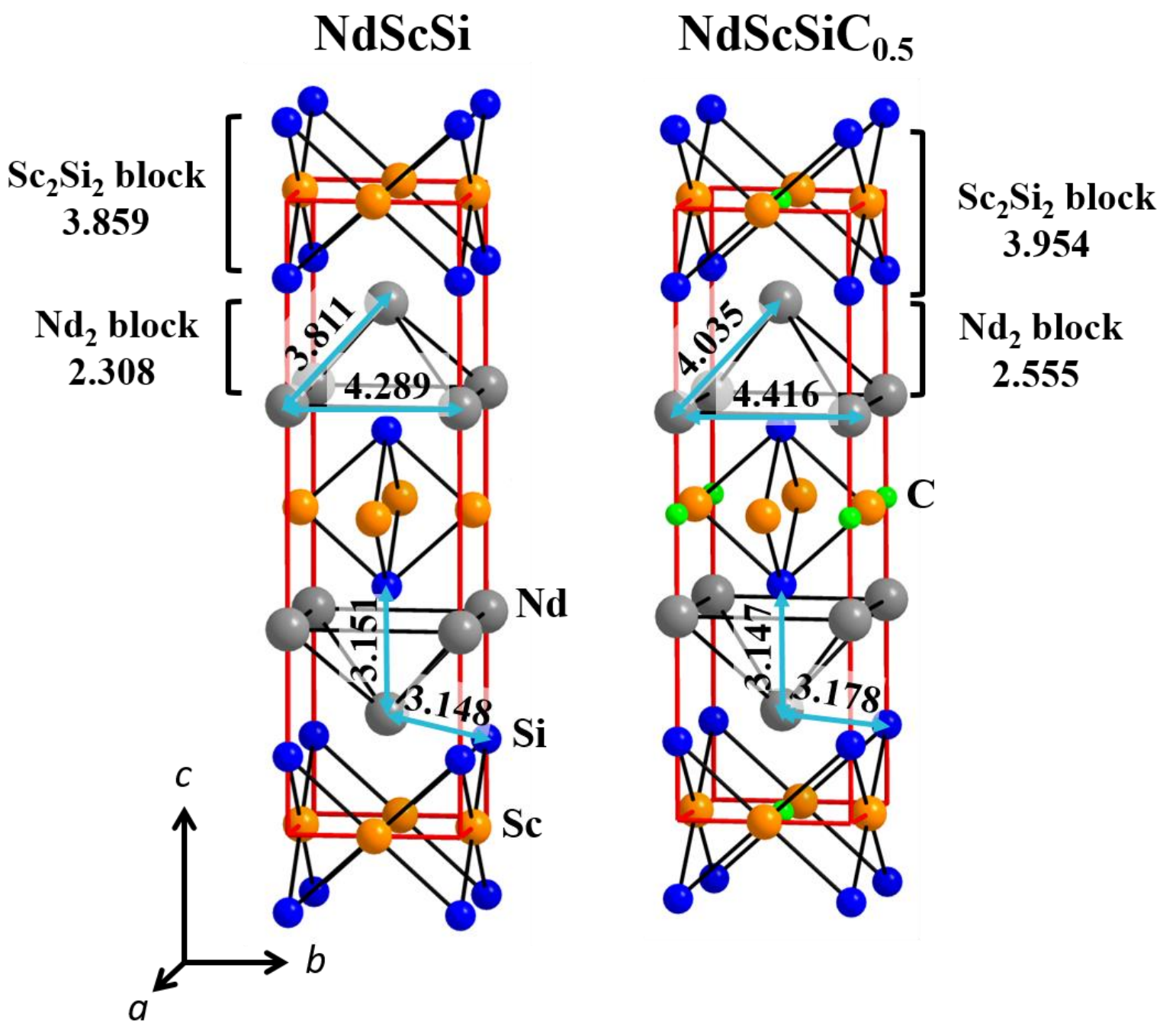

Nd 2 block

2.555

Figure 5. Crystal structures of $\mathrm{NdScSi}$ and $\mathrm{NdScSiC}_{0.5}$ with a drawing of $\mathrm{Nd}-\mathrm{Nd}$ edges of the $\mathrm{Nd}_{4}$ tetrahedra and of Sc-Si bonds. The two couples of $\mathrm{Nd}-\mathrm{Nd}$ and Sc-Si distances are given in angstrom. 
Table 3. Interatomic distances of the $\mathrm{NdScSi}^{7}$ and $\mathrm{NdScSiC}_{0.5}$ compounds. Distances in italics are to the vacant site. Carbide distances are calculated from the single crystal measurements.

\begin{tabular}{|c|c|c|c|}
\hline \multicolumn{2}{|l|}{ Distance } & \multirow{2}{*}{$\begin{array}{l}\text { NdScSi } \\
2.772 \\
\end{array}$} & \multirow{2}{*}{$\begin{array}{l}\mathrm{Nd}_{1.02} \mathrm{Sc}_{0.98} \mathrm{SiC}_{0.5} \\
2.5674(7)\end{array}$} \\
\hline $\mathrm{Nd}-\mathrm{C} / \mathrm{Oh}^{\mathrm{b}}$ & $\times 1$ & & \\
\hline $\mathrm{Nd}-\mathrm{Si}^{\mathrm{a}}$ & $\times 4$ & 3.148 & $3.1778(6)$ \\
\hline $\mathrm{Nd}-\mathrm{Si}^{\mathrm{a}}$ & $\times 1$ & 3.151 & $3.147(2)$ \\
\hline $\mathrm{Nd}-\mathrm{Sc}$ & $\times 4$ & 3.505 & $3.3862(6)$ \\
\hline $\mathbf{N d}-\mathbf{N d}^{\mathrm{a}}$ & $\times 4$ & 3.811 & $4.0350(6)$ \\
\hline $\mathrm{Nd}-\mathrm{Nd}^{\mathrm{a}}$ & $\times 4$ & 4.289 & $4.416(1)$ \\
\hline $\mathrm{Sc}-\mathrm{C} / \mathrm{Oh}^{\mathrm{b}}$ & $\times 2$ & 2.145 & $2.2078(5)$ \\
\hline Sc-Si & $\times 4$ & 2.885 & $2.963(1)$ \\
\hline Sc-Sc & $\times 4$ & 3.033 & $3.1224(5)$ \\
\hline $\mathrm{Si}-\mathrm{C} / \mathrm{Oh}$ & $\times 4$ & 3.595 & $3.695(1)$ \\
\hline
\end{tabular}

${ }^{a}$ See figure $5{ }^{b}$ See figure 6

The interatomic distances for $\mathrm{NdSCSi}$ and $\mathrm{Nd}_{1.02} \mathrm{Sc}_{0.98} \mathrm{SiC}_{0.5}$ from the literature and derived during this work are given in Table 3. Contrary to what was previously observed after deuteriation (or hydrogenation) of the pristine $\mathrm{NdScSi}{ }^{7}$, insertion of carbon induces a decrease of the distance between $\mathrm{Nd}_{2}$ and $\mathrm{Sc}_{2} \mathrm{Si}_{2}$ blocks and an increase of interatomic distances inside them (figure 5). The distance between the middle of each of these two blocks (equal to $c / 4$ ) decreases from $3.926 \AA$ to $3.845 \AA$ for the carbide whereas it increased to 4.158 $\AA$ for the deuteride, showing clearly that carbon insertion reinforces the 3D character of the pristine compound. In the two block-types all the distances increase ( $\mathrm{Nd}-\mathrm{Nd}$, Sc-Si, Sc-Sc, see Table 3). For instance, the new $d_{N d-N d}$ distances equal to 4.035 and $4.416 \AA$ are larger than that seen in Nd metal ( $3.65 \AA$ ) and NdScSi (3.811 and $4.289 \AA$ ), which suggests that the Nd-Nd interactions are reduced in the carbide (figure 5). This different behaviour compared to the deuteride (the later distances are equal to 3.686 and $4.188 \AA$ ) seems to be directly correlated to the $\mathrm{Nd}-\mathrm{C}$ bonding in the $\mathrm{Sc}_{4} \mathrm{Nd}_{2}$ octahedral site. In NdScSi the distance between the $2 b$ site (occupied by $\mathrm{C}$ in the carbide) and the neighboring $\mathrm{Nd}$, equal to $2.77 \AA$, is reduced to $2.57 \AA$ $\left(=d_{N d-C}\right)$ in the carbide (figure 6$)$. This is achieved by a decrease of the $c$ parameter $(-2.1 \%$, Table 1) and a slight shift of $\mathrm{Z}_{\mathrm{Nd}}$ coordinate. This latter distance is comparable to the values observed for the two binary Nd carbides: $\mathrm{NdC}_{2}{ }^{35}$ and $\mathrm{Nd}_{2} \mathrm{C}_{3}{ }^{36}$ where $\mathrm{d}_{\mathrm{Nd}-\mathrm{C}}=2.553 \AA$ and 2.611 $\AA$ respectively. Moreover the filling of the Sc square plane by carbon induces an increase of the $a$ parameter to manage more realistic Sc-C distances from 2.145 in the pristine to 2.208 $\AA$ in $\mathrm{Nd}_{1.02} \mathrm{Sc}_{0.98} \mathrm{SiC}_{0.5}$. This distance is comparable to the ones observed in $\mathrm{ScC}^{37}, \mathrm{Sc}_{3} \mathrm{C}_{4}{ }^{38}, \mathrm{Sc}_{4} \mathrm{C}_{3}$ 
${ }^{39}$, and $\mathrm{SC}_{15} \mathrm{C}_{19}{ }^{40}$ for which the Sc-C distances range between 2.25 and $2.35 \AA$. The opposite evolution of cell parameters after carbon insertion when compared to hydrogen insertion is then explained by the strong covalent bonding between carbon and surrounding $\mathrm{Nd}$ and Sc atoms forming the octahedral site. The drawing of the $\mathrm{Sc}_{4} \mathrm{Nd}_{2}$ octahedra filled by carbon atoms shows that $\mathrm{Nd}_{1.02} \mathrm{Sc}_{0.98} \mathrm{SiC}_{0.5}$ crystallizes in an anti- $\mathrm{K}_{2} \mathrm{NiF}_{4}$ structure (figure 6 ). In the following, the $\mathrm{NdScSiC} 0.5$ formula omitting the $\mathrm{Nd} / \mathrm{Sc}$ mixing will be used for simplicity.
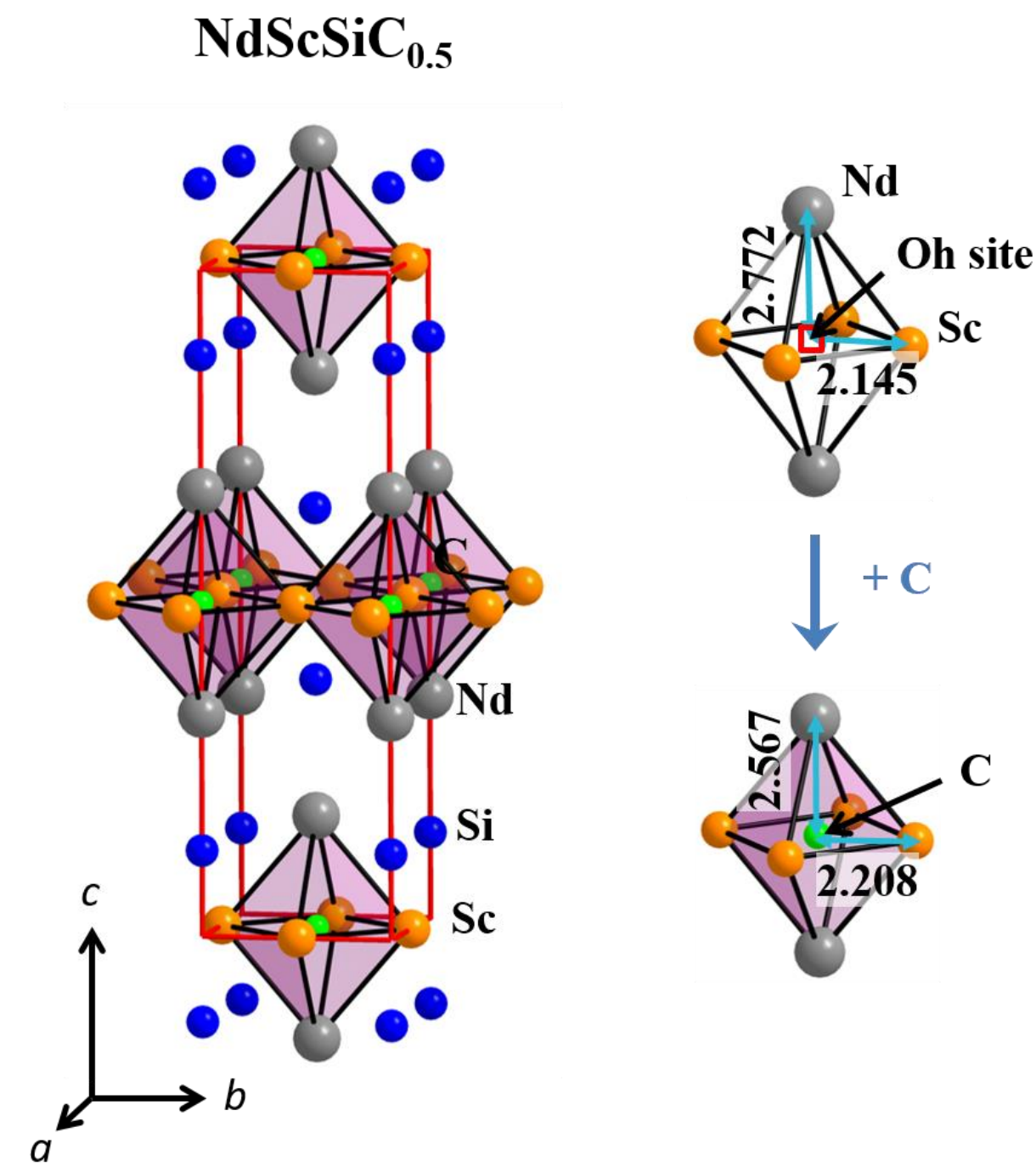

Figure 6. Left: Crystal structures of $\mathrm{NdScSiC}_{0.5}$ with the drawing in purple of the $\mathrm{Sc}_{4} \mathrm{Nd}_{2}$ octahedra filled by $\mathrm{C}$ atoms. Right: Environment of the octahedral site before ( $\mathrm{NdScSi}^{7}$ ) and after insertion of carbon atom $\left(\mathrm{NdScSiC}{ }_{0.5}\right)$. The distances between the center of the octahedral site and $\mathrm{Nd}$ or Sc atoms are given in angstrom. 


\section{Magnetization measurements}
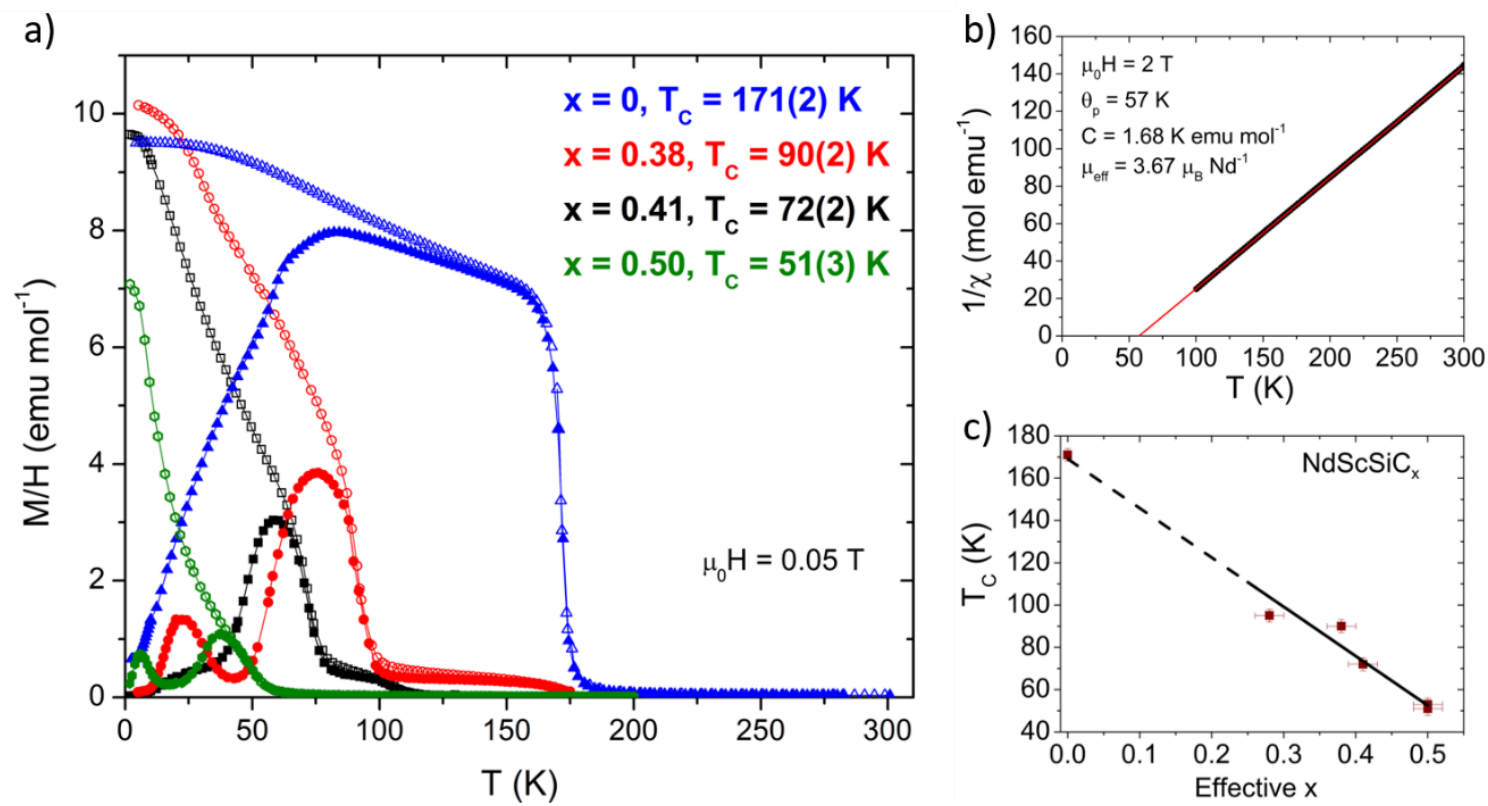

Figure 7. (colour online) a) Magnetization curves $M / H(T)$ for the $N d S c S i C_{x}$ for effective $x=$

$0,0.38,0.41$ and 0.50 at $0.05 \mathrm{~T}$. The filled symbols show the zero-field cooled (ZFC) measurement and the hollow ones the field cooled (FC) measurement. b) Temperature dependence of the inverse of the magnetic susceptibility at $\mu_{0} H=2 \mathrm{~T}$ for $\mathrm{NdScSiC}_{0.5}$ fitted using the Curie-Weiss law. c) Evolution of the Curie temperature of the $\mathrm{NdScSiC}_{\mathbf{x}}$ compounds with respect to the calculated effective carbon content.

Figure 7 (b) shows a plot of the inverse susceptibility against temperature for $\mathrm{NdScSiC}_{0.5}$ in a high field of $2 \mathrm{~T}$, fitted using the Curie-Weiss law. $\mathrm{NdScSiC}_{0.5}$ is a Curie-Weiss paramagnet with a paramagnetic Curie temperature $\left(\theta_{\mathrm{P}}\right)$ of $57 \mathrm{~K}$. The positive value of $\theta_{\mathrm{P}}$ indicates predominant ferromagnetic local interactions and the value is close to the measured Curie temperature of around $50 \mathrm{~K}$. The calculated effective moment of $3.67 \mu_{\mathrm{B}} \mathrm{Nd}^{-1}$ corresponds very well with the $3.62 \mu_{\mathrm{B}}$ for the free $\mathrm{Nd}^{3+}$ ion. From this, it can be concluded that only the rare earth atoms are magnetic in these compounds.

$\mathrm{NdScSi}$ compound is known to be a ferromagnet with a $T_{C}$ of $171 \mathrm{~K}^{41}$. Zero-field-cooled and field-cooled magnetization against temperature curves are depicted in Figure 7 (a) for a selection of samples from the the $\mathrm{NdScSiC}_{x}$ solid solution. These measurements show that the carbides display ferromagnetic order. However, carbon insertion causes a reduction in the $T_{C}$ (determined from the inflection point of the (ZFC) curves) of the system with increasing carbon content from $171 \mathrm{~K}$ for the pristine $\mathrm{NdScSi}$ ( ${ }^{41}$ and this work) to approximately $50 \mathrm{~K}$ for $\mathrm{NdScSiC}{ }_{0.5}$. From the curves shown in Figure 7 (a) there is a clear divergence between the ZFC and FC parts of the measurement. This is often observed in rare earth based magnetic compounds and stems from their generally large magnetocrystalline anisotropy ${ }^{41}$. Several small magnetic anomalies, associated with secondary phases, can be observed in the $M(T)$ 
curves for $x=0.38, x=0.41$ and $x=0.50$ plots (Figure $7(a)$ ). For $x=0.50$, the anomaly around $10 \mathrm{~K}$ is due to the $\mathrm{NdSi}_{2-\mathrm{x}}$ secondary phase which is reported to be ferromagnetic at $T_{C} \approx 10 \mathrm{~K}$ in the literature ${ }^{42}$. As illustrated in Figure S2, the amount of this silicide rises by increasing the nominal carbon content up to 0.7 , i.e. when moving away from the maximal $x=0.5$ effective value that can be reached in $\mathrm{NdScSiC}_{\mathrm{x}}$. In the $\mathrm{x}=0.41$ curve a magnetic anomaly is observed at $115 \mathrm{~K}$ and can be attributed to residual $\mathrm{Nd}_{5} \mathrm{Si}_{3}$ which is found to be ferromagnetic at $T_{C} \approx 115 \mathrm{~K}$ by Boulet et al. ${ }^{42}$. Finally, a very small amount of residual $\mathrm{NdSCSi}$ is also visible in the $x=0.38$ curve that could not be observed from $X$-ray diffraction alone on this sample. The transition around $25 \mathrm{~K}$ in the other $\mathrm{x}=0.38$ sample could not be identified. As other samples do not show this transition it is not intrinsic to the major phase and thus must belong to a secondary phase, possibly to the $\mathrm{Nd}_{2} \mathrm{ScSi}_{2}$ phase observed in the XRD pattern of this sample. As the magnetic properties of this phase have not been previously documented this cannot be confirmed here. Finally, from the plot of the Curie temperature of the $\mathrm{NdScSiC}_{x}$ carbides against the effective carbon content $x$ (Figure 7 (c)) it is clear that the $T_{C}$ decreases linearly with $x$. Extrapolation of the linear fit of the data points outside of the solid solution domain also intersects the pristine NdScSi. Therefore, carbon insertion could potentially be used as a method of tuning the magnetic ordering temperatures of CeScSi-type compounds in the future.

Isothermal magnetization measurements, $\mathrm{M}(\mathrm{H})$, of $\mathrm{NdScSiC}_{0.50(2)}$ for several temperatures between 1.8 and $120 \mathrm{~K}$ are shown in Figure 8 (left). To reduce the effects of magnetocrystalline anisotropy these measurements were performed on powder samples. The sharp increase in magnetization with field confirms the ferromagnetic ground state of the carbide. At $120 \mathrm{~K}$ the linearity of the curve is as expected for a paramagnetic state, thus no residual $\mathrm{NdScSi}$ remains in this sample. At $7 \mathrm{~T}$ and low temperatures, the magnetization appears to be almost saturated at $2.67 \mu_{\mathrm{B}} \mathrm{f.u} .^{-1}$, significantly below the value expected for a free ion $\mathrm{Nd}^{3+}$ moment $\left(3.27 \mu_{\mathrm{B}}\right.$ ). The reduced magnetization at $7 \mathrm{~T}$ is likely due to a combination of the magnetocrystalline anisotropy and a splitting of the ground state by the crystal electric field reducing the magnetic moment. 

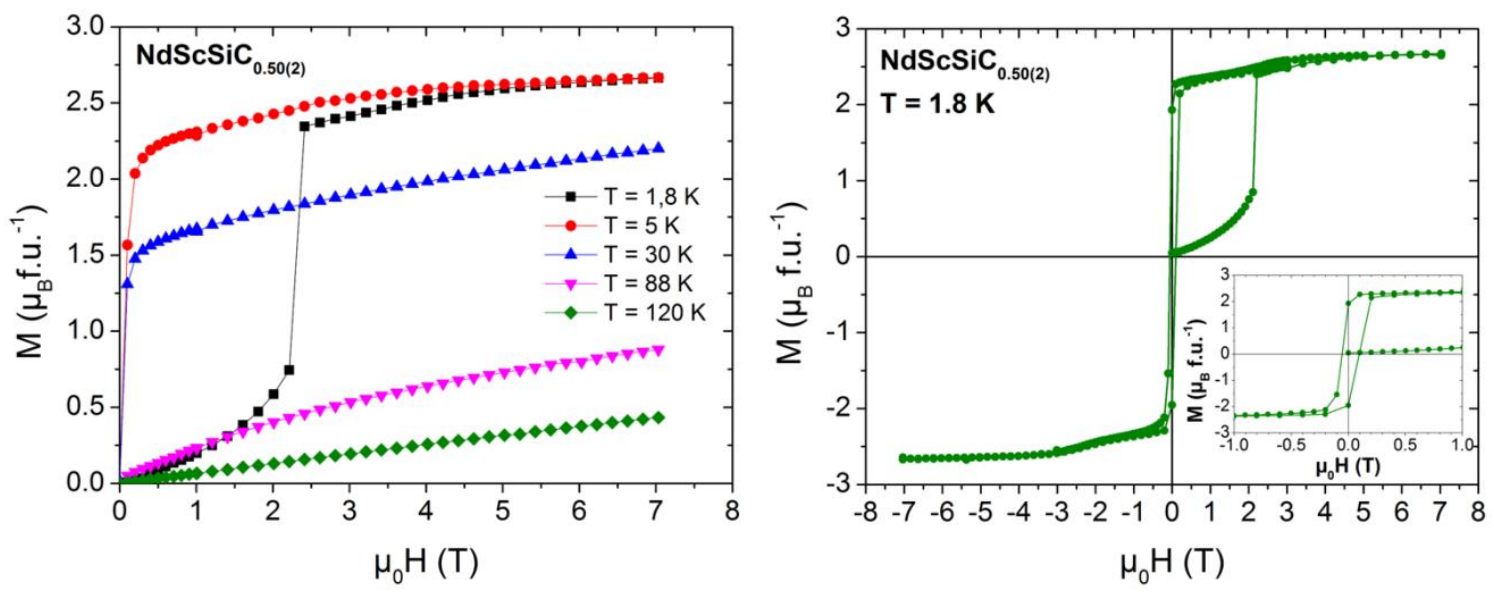

Figure 8. (Left): Isothermal magnetization curves $M(\mathrm{H})$ for $\mathrm{NdScSiC}_{0.50(2)}$ (effective composition) powder at various temperatures between 1.8 and $120 \mathrm{~K}$ in fields of up to $7 \mathrm{~T}$. The curve at $1.8 \mathrm{~K}$ represents a virgin magnetization curve. (Right): Isothermal hysteresis cycle of a powder sample at $1.8 \mathrm{~K}$ between +7 and $-7 \mathrm{~T}$ for $\mathrm{NdScSiC}_{0.50(2)}$ (left) and a zoom on the region 1 to $-1 \mathrm{~T}$ (right). The measurement was performed on a powder sample. Inset: zoom of the low field region.

Figure 8 (right) shows the complete cycle of the isothermal magnetization of a $\mathrm{NdScSiC}_{0.50(2)}$ powder sample at $1.8 \mathrm{~K}$ in the field range of +7 to $-7 \mathrm{~T}$. The virgin magnetization curve of the material begins with a gradual increase of the magnetization with a sudden, sharp jump around $2.3 \mathrm{~T}$, almost reaching the saturation magnetization. This behavior suggests a hard ferromagnet with slow movement of the domain walls. This is likely due to the presence of magnetocrystalline anisotropy and defects that pin the domain walls and requires an increase in the field to force their displacement. The presence of large magnetocrystalline anisotropy is in agreement with the separation between the ZFC and FC curves in Figure, as previously observed in the pristine $\mathrm{NdScSi}$ by Singh et al. ${ }^{41}$. However, the domain wall pinning appears weaker in $\mathrm{NdScSi}$ and the saturation is more easily reached. Furthermore, the hysteresis curve shows that, after initial magnetization, $\mathrm{NdScSiC}_{0.50(2)}$ has a significant remnent magnetization of $1.93 \mu_{\mathrm{B}} \mathrm{Nd}^{-1}$ but a reasonably small coercive field of roughly $0.05 \mathrm{~T}$ as expected for a powder sample.

\section{Neutron diffraction}

Neutron diffraction measurements were performed on both $\mathrm{NdScSi}(1.5-200 \mathrm{~K})$ and $\mathrm{NdScSiC} \mathrm{C}_{0.5}$ $(1.5-100 \mathrm{~K})$ to investigate and compare their magnetic structures. These temperature ranges were chosen based on the ZFC-FC magnetization curves shown in Figure 7 (a) (NdScSi) and Figure 9 (left) ( $\mathrm{NdScSiC}_{0.5}$ ). The latter of which shows two magnetic ordering phenomena. The first, around $110 \mathrm{~K}$ is attributed to the secondary phase $\mathrm{Nd}_{5} \mathrm{Si}_{3}{ }^{42}$ observed in the XRD pattern for this sample, the second transition at $79 \mathrm{~K}$ is therefore expected to be attributed to $\mathrm{NdScSiC}_{0.5}$. However, by Figure 7 (c) the $T_{C}$ corresponds to an effective $\mathrm{x}$ of approximately 0.40 . This can be explained by a more careful consideration of the XRD pattern for this sample. Figure 9 (right) shows the (006) peak of the XRD pattern for this sample. On careful 
examination we observe that, rather than a single peak around $2 \theta=34.9^{\circ}$, this peak is formed of a number of strongly overlapping peaks, each corresponding to a different $c$ parameter (as labelled). The approximate $c$ parameter for each of these peaks was calculated and, as well as the two labelled $c$ parameters ( 15.3948 and $15.4122 \AA$ ) that yield effective $x$ values of $0.48(2)$ and $0.44(2)$ respectively, there is an additional small shoulder at lower angles. This peak is indexed with $c=15.4428 \AA$ which corresponds to $x=0.39(2)$ (Figure 3), in good agreement with the observed $T_{C}$ (by Figure 7 (c)). Thus the carbon is inhomogeneously distributed throughout the sample which is likely due to the large sample volume needed for neutron diffraction. The refinement of the nuclear and magnetic structure (discussed later in Figure 11 and Table 4) indicates the average composition is $\mathrm{NdScSiC}_{0.47(1)}$, i.e. very close to the target value of $\mathrm{NdScSiC}_{0.5}$. Therefore, the broadened ferromagnetic transition, ascribed to some distribution of the carbon content between $\mathrm{x}=0.39$ and 0.48 , arises below $T_{C}=79 \mathrm{~K}$, Curie temperature which corresponds to the least carbon rich part of the sample.
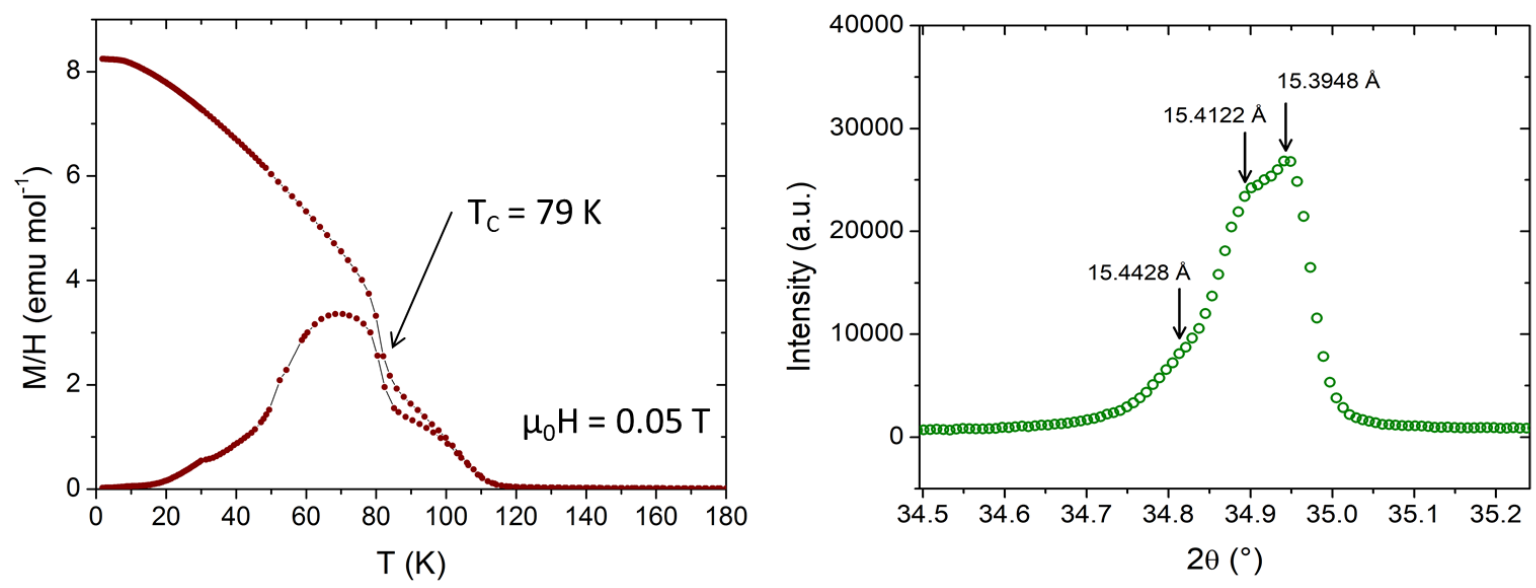

Figure 9 (Left): Magnetic susceptibility against temperature for the large (5 g) neutron sample of $\mathrm{NdScSiC}_{0.47(1)}$ in a field of $0.05 \mathrm{~T}$. The filled points so the ZFC curve and the hollow points show the FC curve. The transition at $115 \mathrm{~K}$ is due to $\mathrm{Nd}_{5} \mathrm{Si}_{3}$ secondary phase. (Right): The (006) peak from high resolution XRD of this sample with the three $c$ parameters labelled.

The neutron diffraction patterns for both compounds at $1.5 \mathrm{~K}$ and above their respective $T_{C}$ are given in Figure 10. The difference patterns emphasise clearly the magnetic peaks. In the case of the pristine $\mathrm{NdScSi}$ an anisotropic contraction of the unit cell is observed on cooling from $180 \mathrm{~K}$ to $1.5 \mathrm{~K}$, with the $a$ parameter decreasing from 4.2817 (7) $\AA$ to $4.2674(2) \AA$ (-0.33 $\%)$ and $c$ increasing slightly from $15.672(3) \AA$ to $15.706(1) \AA(+0.22 \%)$. This reduces the unit cell volume from $287.3 \AA^{3}$ to $286.0 \AA^{3}$. Meanwhile, on cooling of the sample $\mathrm{NdScSiC} \mathrm{C}_{0.5}$ from $100 \mathrm{~K}$ to $1.5 \mathrm{~K}$, the unit cell parameter $a$ shrinks from 4.41298 (5) $\AA$ to 4.39676 (7) $\AA$ (-0.37\%) while $c$ remains almost constant from $c=15.3825(2) \AA$ to $15.3890(6) \AA$ (+0.04\%), leading to a total change in the unit cell volume of $-0.70 \%$. 

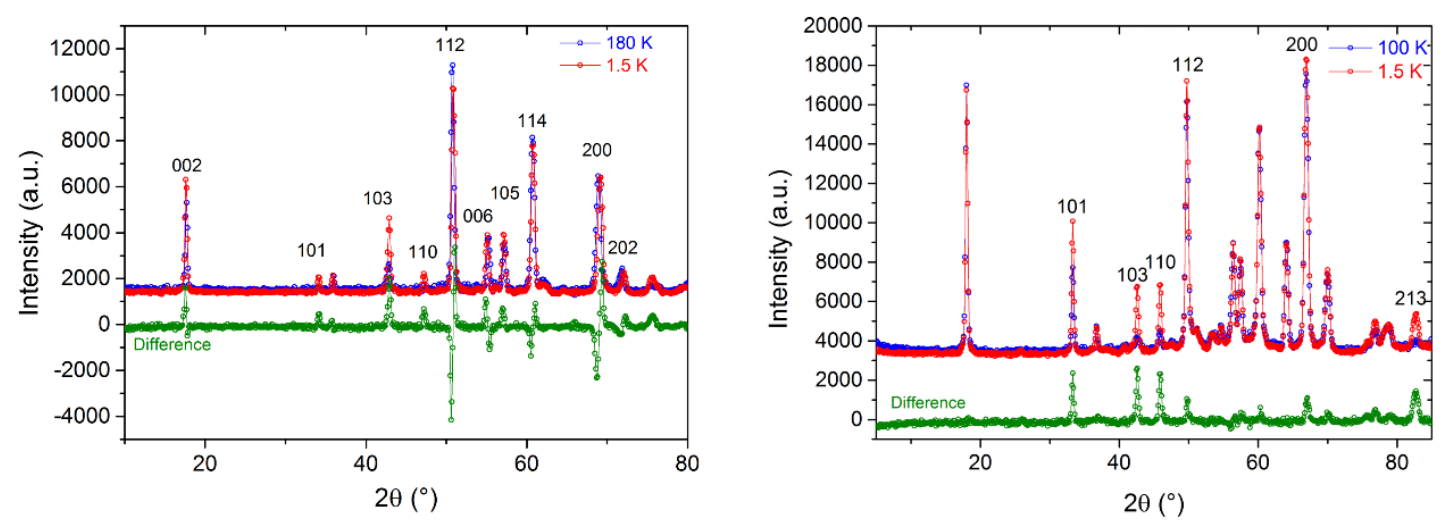

Figure 10. Neutron diffraction patterns at $1.5 \mathrm{~K}$ and above the $\mathrm{T}_{\mathrm{c}}$ of $\mathrm{NdScSi}$ (left) and $\mathrm{NdScSiC}_{0.5}$ (right) with the difference patterns shown below.

In $\mathrm{NdScSi}$ and $\mathrm{NdScSiC}_{0.5}$ compounds the magnetic peaks coincide with the nuclear peaks, meaning that they are indexed with the propagation vector $k=(000)$ and confirming that the magnetic and crystalline unit cells are the same. Both data were refined using a ferromagnetic structure. For $\mathrm{NdScSiC}_{0.5}$ the absence of magnetic contributions on the (0Ol) lines indicate that the magnetic moments are aligned along the $c$-axis. This collinear ferromagnetic structure is confirmed by the magnetic Rietveld refinement at $1.5 \mathrm{~K}$. The situation is different for $\mathrm{NdScSi}$, where the presence of magnetic signal on the (OOl) lines suggests the existence of a magnetic moment component within the ab-plane. As there is only one $\mathrm{Nd}$ position, the magnetic structure could be unambiguously determined and the solution corresponds to magnetic moments aligned only in the $a b$-plane (directions in the $a b$-plane are equivalent from powder neutron diffraction for a tetragonal structure). Attempts to include a component along the $c$ axis worsened the fit significantly. The refinement at $1.5 \mathrm{~K}$ for both compounds is shown in Figure 11 and a summary of the refined parameters is given in Table 4. The magnetic structures of $\mathrm{NdScSi}$ and $\mathrm{NdScSiC} 0.5$ are shown in Figure 12. Carbon insertion causes a change in the easy axis of magnetization while conserving a ferromagnetic coupling. It is noteworthy that the substitution of Si by Ge also provokes a reorientation of the magnetic moments since they are aligned with an angle of $54^{\circ}$ with the $c$-axis in the ferromagnet $\mathrm{NdScGe}\left(T_{C}=200 \mathrm{~K}\right)^{43}$. The refined magnetic moment of the $\mathrm{NdScSi}$ compound $\left(2.38(5) \mu_{\mathrm{B}} \mathrm{Nd}^{-1}\right.$ ) at $1.5 \mathrm{~K}$ is significantly below the free ion saturation value expected for a $\mathrm{Nd}^{3+}\left(3.27 \mu_{\mathrm{B}}\right)$. However this is in good agreement with magnetization measurements carried out at $5 \mathrm{~K}$ on NdScSi by Singh et al. ${ }^{41}$ which indicated a reduction in the magnetic moment to about $2.0 \mu_{\mathrm{B}} \mathrm{Nd}^{-1}$ due to strong magnetocrystalline anisotropy. Carbon insertion clearly reduces this effect, leading to an increase in the refined saturated moment to 3.09(4) $\mu_{B} \mathrm{Nd}^{-1}$ which is slightly higher than the $2.7 \mu_{\mathrm{B}} \mathrm{f} . \mathrm{u} .^{-1}$ found in the isothermal magnetization measurements at $7 \mathrm{~T}$, indicating that the sample was not fully magnetically saturated at this field. 

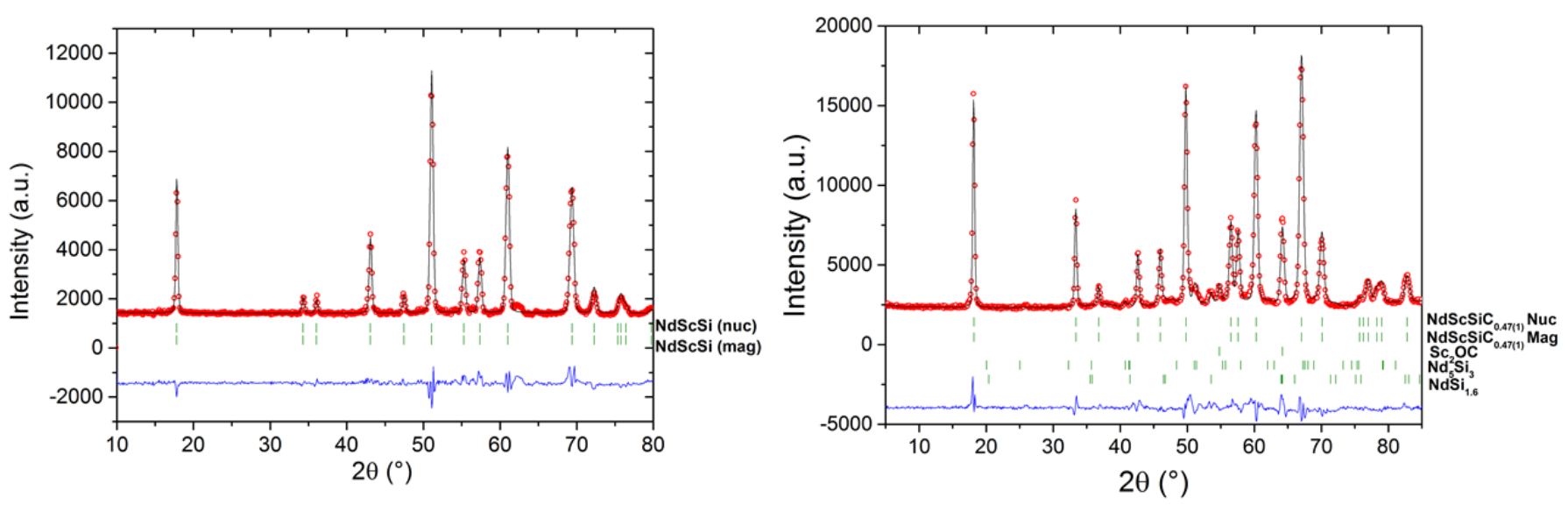

Figure 11. Rietveld refinements of the magnetic structures of $\mathrm{NdScSi}$ (left) and $\mathrm{NdScSiC} \mathrm{C}_{0.5}$ (right) at $1.5 \mathrm{~K}$.

Table 4. Summary of the refined parameters of the nuclear and magnetic structure of $\mathrm{NdScSi}$ and $\mathrm{NdScSiC}(0.47(1)$ at $1.5 \mathrm{~K}$.

\begin{tabular}{|c|c|c|c|c|c|c|c|c|c|}
\hline Phase & $a(\AA ̊)$ & $c(\AA ̊)$ & $\begin{array}{l}\text { Nd } \\
\text { Moment } \\
\left(\mu_{B}\right)\end{array}$ & $\overline{z_{\mathrm{Nd}}}$ & $\overline{z_{\mathrm{Si}}}$ & $\operatorname{Occ}(C)$ & $\begin{array}{l}R- \\
\text { Bragg } \\
(\%) \\
\end{array}$ & $\begin{array}{l}R_{P} \\
(\%)\end{array}$ & $\begin{array}{l}R_{w} \\
(\%)\end{array}$ \\
\hline $\begin{array}{l}\text { NdScSi } \\
\text { Nuclear } \\
\text { NdScSi } \\
\text { Magnetic }\end{array}$ & $4.2674(2)$ & $15.706(1)$ & $\begin{array}{l}- \\
2.38(5)\end{array}$ & $0.3228(5)$ & $0.124(1)$ & - & $\begin{array}{l}8.5 \\
11.5\end{array}$ & 20.5 & 15.7 \\
\hline $\begin{array}{l}\text { NdScSiC }_{0.5} \\
\text { Nuclear } \\
\text { NdScSiC }_{0.5} \\
\text { Magnetic }\end{array}$ & $4.3967(1)$ & $15.3886(8)$ & $\begin{array}{l}- \\
3.09(4)\end{array}$ & $0.333(2)$ & $0.1279(8)$ & $0.94(2)$ & $\begin{array}{l}5.1 \\
5.7\end{array}$ & 15.2 & 13.1 \\
\hline
\end{tabular}



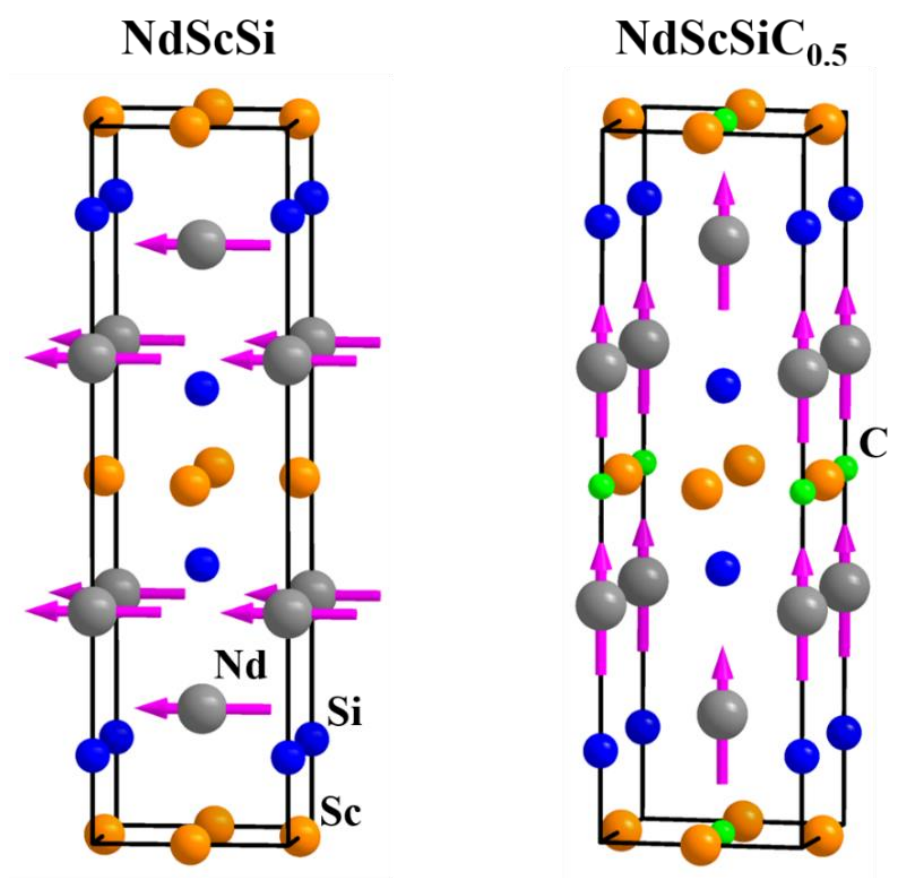

Figure 12. Magnetic structures of $\mathrm{NdScSi}$ and $\mathrm{NdScSiC}_{0.5}$ at $1.5 \mathrm{~K}$. The magnetic moments are arbitrarily represented along the $a$-axis for $\mathrm{NdScSi}$.

The evolution of the refined $\mathrm{Nd}$ magnetic moments of $\mathrm{NdScSi}$ and $\mathrm{NdScSiC}_{0.47(1)}$ with temperature (Figure 13) were both determined using the same ferromagnetic model over the whole temperature range. This confirmed $T_{C}$ temperatures around 170 and $80 \mathrm{~K}$ respectively and the absence of other magnetic transitions at lower temperatures such as spin reorientation. For $\mathrm{NdScSiC}_{0.47(1)}$ no change in the intensity of the main phase peaks was observed above $80 \mathrm{~K}$, confirming our hypothesis that the magnetic transition observed around $110 \mathrm{~K}$ is due to the $\mathrm{Nd}_{5} \mathrm{Si}_{3}$ secondary phase. These results demonstrate that the type of magnetic order is maintained through carbon insertion in NdScSi but the crystal electric field effect is strongly modified as evidenced by the change of the easy axis of magnetization and the magnetic moment value.
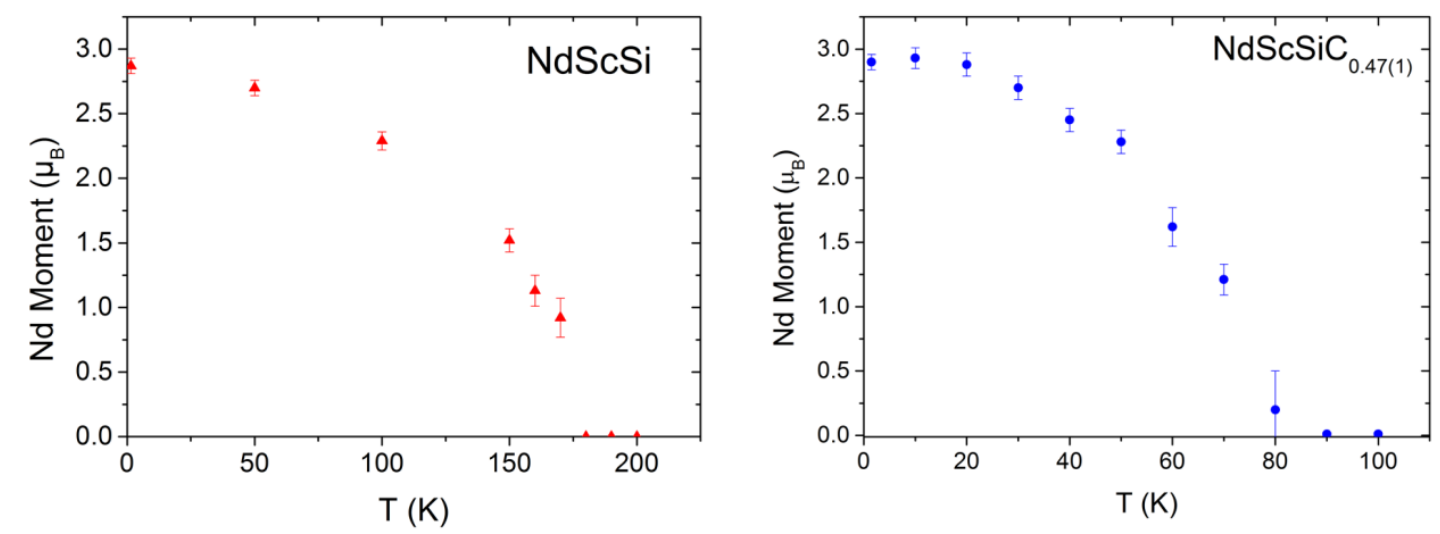

Figure 13. Refined Nd moment versus temperature for $\mathrm{NdScSi}$ (left) and $\mathrm{NdScSiC} \mathbf{0}_{0.47(1)}$ (right). 


\section{DFT calculations}

We performed DFT calculations for the three compounds $\mathrm{NdScSi}, \mathrm{NdScSiC} 0.5$ and $\mathrm{NdScSiH}_{1.5}$ to compare their electronic structures, spin densities and chemical bonding features. For the hydride $\mathrm{NdSCSiH}_{1.5}$ we note $\mathrm{H} 1$ and $\mathrm{H} 2$ hydrogen atoms located respectively at the $4 d(0 \mathrm{r} / 2$ $1 / 4)$ and $4 e\left(\begin{array}{lll}0 & 0 & 0.518\end{array}\right)$ positions in the unit cell (S.G. $14 / \mathrm{mmm}, 4$ formula units). ${ }^{7} \mathrm{H} 1$ lies in a $\mathrm{Nd}_{4}$ tetrahedral site; $\mathrm{H} 2$ lies off-centered in a $\mathrm{Sc}_{4} \mathrm{Nd}_{2}$ octahedral site, with apical $\mathrm{H} 2-\mathrm{Nd}$ bonds of 2.60 and $3.16 \AA$ with $\mathrm{Nd}$ atoms of different $\mathrm{Nd}$ double layers, along the $c$-axis.

Figure 14 shows the calculated densities of states (DOS) for $\mathrm{NdScSi}, \mathrm{NdScSiC} 0.5$ and $\mathrm{NdScSiH}_{1.5}$, with several atomic contributions. $\mathrm{Nd} 4 \mathrm{f}$ partial DOS are readily identified as pronounced peaks, very narrow (localized states at $4.7 \mathrm{eV}$ below Fermi level $\left(E_{F}\right)$ ) for occupied $4 \mathrm{f}$ states in $\mathrm{NdScSi}$ and $\mathrm{NdScSiH}_{1.5}$. Interestingly, in $\mathrm{NdScSiC}_{0.5}$ the up-spin occupied $4 \mathrm{f}$ bands are significantly broadened and destabilized by up to $1.5 \mathrm{eV}$, indicating strong $\mathrm{C}-\mathrm{Nd}(4 \mathrm{f})$ interactions. The other $\mathrm{Nd}$ contributions to the valence bands arise from $\mathrm{Nd} 5 \mathrm{~d}$ orbitals, hybridized to Sc $3 d$ orbitals, as seen from the perfectly corresponding DOS; therefore, there are also strong $\mathrm{Nd}$-Sc interactions via d atomic orbitals. $\mathrm{Nd} / \mathrm{Sc} d$-character bands are neatly spin-polarized in $\mathrm{NdScSi}$, whereas there is no such polarization in $\mathrm{NdScSiC}_{0.5}$ and $\mathrm{NdScSiH}_{1.5}$. The carbon DOS contribution spreads all over the valence bands of $\mathrm{NdScSiC}_{0.5}$, while distinct bands are attributed to $\mathrm{H} 1$ and $\mathrm{H} 2$, below $4 \mathrm{f}$ occupied bands of the hydride. Those low-lying $\mathrm{H}$-character bands (in both spin channels) indicate $\mathrm{H}^{-}$species, as expected here; besides, the partial DOS show strong covalent $\mathrm{Sc}-\mathrm{H} 2$ and $\mathrm{Nd}-\mathrm{H} 1$ bonding, leading to a significant shift of $\mathrm{Sc}$ $3 \mathrm{~d}$ spectral weight from $\mathrm{Nd}$-Sc hybridized states in $\mathrm{NdScSi}$ toward $\mathrm{Sc}-\mathrm{H} 2$ and $\mathrm{Nd}-\mathrm{H} 1$ states in the hydride.

As an indicator of the respective magnitudes of the $\mathrm{Nd}-\mathrm{Sc}, \mathrm{Nd}-\mathrm{C}$ and $\mathrm{Nd}-\mathrm{H}$ coupling, we examined the product of atom- or orbital-projected DOS vs. energy, for the valence bands. The DOS product has some similarities with so-called crystal orbital overlap or Hamiltonian populations (COOP and COHP, respectively); however, contrary to those two quantities, the DOS product does not give indications on the bonding $v s$. antibonding nature of the electron states with respect to a selected bond in the crystal. The DOS products provide a qualitative hint on the inter-atomic interactions; whenvever electrons are described by a crystal orbital with contributions of both $\mathrm{Nd}$ (or either $5 \mathrm{~d}$ or $4 \mathrm{f} \mathrm{Nd}$ orbitals) and a neighbor atom (Sc, C, $\mathrm{H}$...), the DOS product will be significant. Also, integrating the DOS product up to $E_{F}$ (or examining the respective areas of DOS product vs. energy peaks) allows discussing qualitatively the magnitude of the magnetic coupling between atomic species or orbitals. 

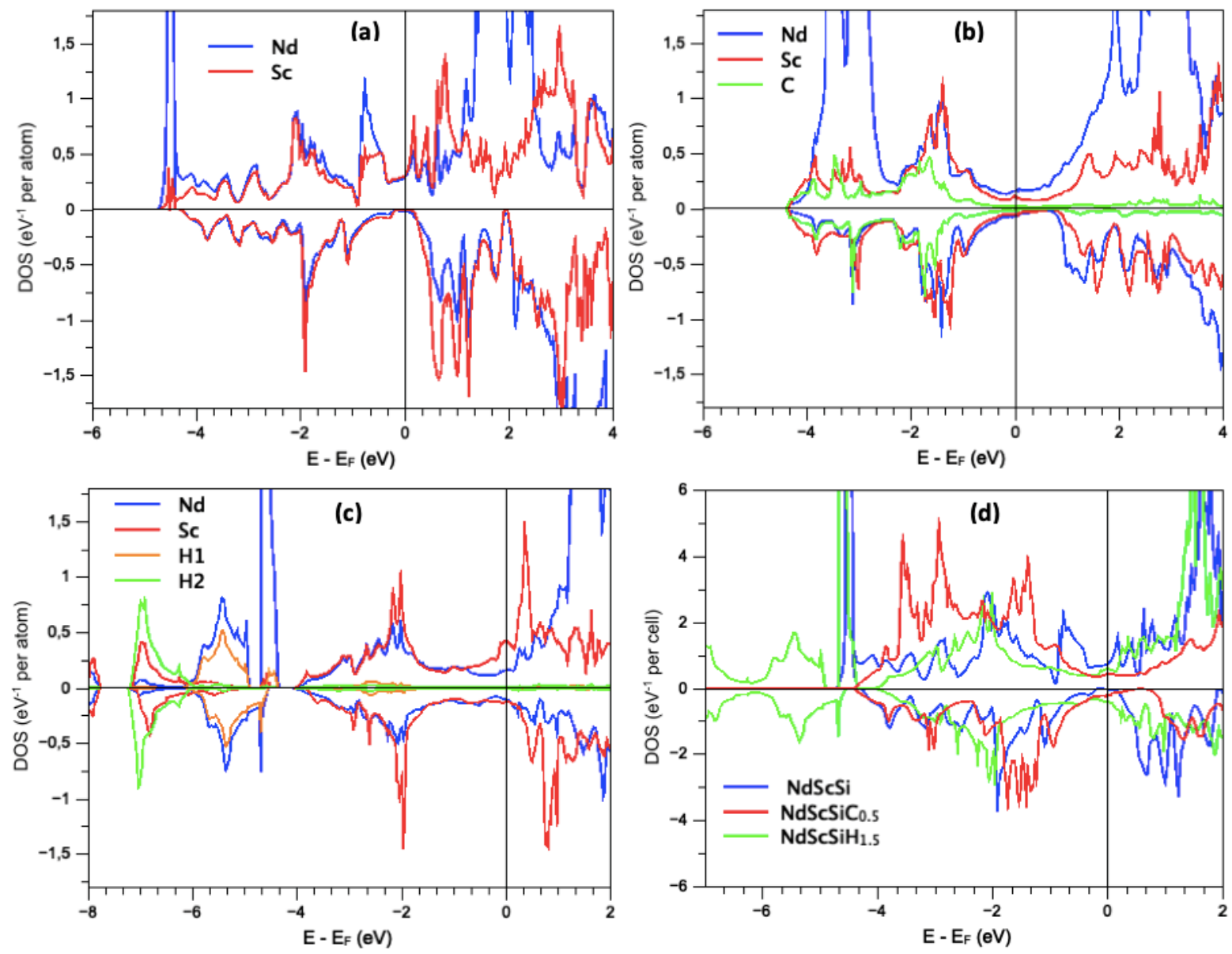

Figure 14. Some projected densities of states for (a) $\mathrm{NdScSi}$, (b) $\mathrm{NdScSiC}_{0.5}$ and (c) $\mathrm{NdScSiH}_{1.5}$, calculated from DFT/HSE06. (d) Total density of states for the three compounds.

First, let us examine the $\mathrm{Nd} 5 \mathrm{~d}$ vs. $\mathrm{Nd} 4 \mathrm{f}$ orbitals contribution to the Nd-Sc DOS products vs. energy, shown in Figure 15. 5d-3d interactions are largely dominant in NdScSi and strongly reduced in $\mathrm{NdScSiH}_{1.5}$, while both $5 \mathrm{~d}$-3d and up-spin $4 \mathrm{f}-3 \mathrm{~d}$ interactions are significant in $\mathrm{NdScSiC}_{0.5}$; a key feature here in the latter compound is the destabilization and huge broadening of the up-spin $4 \mathrm{f}$-character band.

In $\mathrm{NdScSi}$, the $5 \mathrm{~d}-3 \mathrm{~d}$ product is significant all over the valence bands, especially for the majority spin channel that has an extra peak in the 1-eV range below $E_{F}$. In the carbide, the overall picture is different with the latter peak shifted by $1 \mathrm{eV}$ away from $E_{F}$. In other words, the $\mathrm{Nd}(5 \mathrm{~d})-\mathrm{Sc}(3 \mathrm{~d})$ DOS product remains significant in $\mathrm{NdScSiC}_{0.5}$ but is no longer spinpolarized, contrary to the $5 \mathrm{~d}-3 \mathrm{~d}$ product in $\mathrm{NdScSi}$. In agreement with the high Curie temperatures observed in $\mathrm{NdScSi}\left(T_{C}=175 \mathrm{~K}\right)$ and $\mathrm{NdScSiC}_{0.5}\left(T_{C} \approx 50 \mathrm{~K}\right)$, the areas of the DOS product peaks are similar in the two compounds. Also, in agreement with the disappearance of magnetic ordering upon hydrogen insertion, the magnitude of the DOS products is significantly reduced in $\mathrm{NdScSiH}_{1.5}$. In our recent works on $\mathrm{GdScGe}$ and $\mathrm{GdScGeH}$, we concluded that the disappearance of magnetic ordering in the hydride was partly due to the weakening of $\mathrm{Gd}-\mathrm{Sc}$ interactions by the competing $\mathrm{Gd}-\mathrm{H} 1$ covalent bonding. ${ }^{6}$ This argument 
seems to hold in $\mathrm{NdScSi}$, with the destruction of the magnetic ordering of the rare earth double layers upon hydrogen insertion.
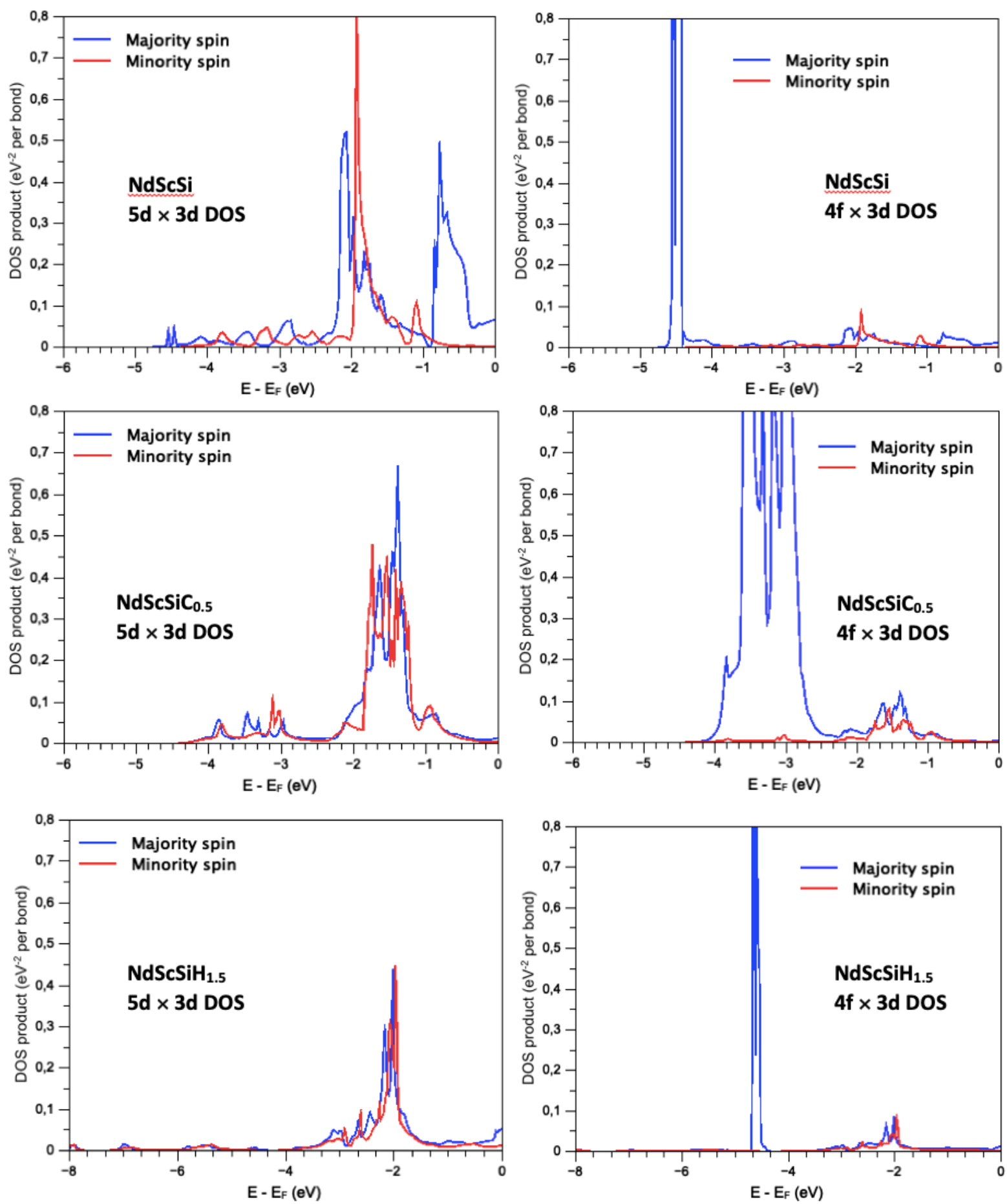

Figure 15. Left: $5 d(N d)-3 d(S c)$ DOS products vs. energy (with respect to Fermi level), calculated from DFT/HSE06. Right: $4 f(N d)-3 d(S c)$ DOS products vs. energy (with respect to Fermi level).

As seen in Figures 14 (b), 15 and 16 (c), the broadening of the $4 \mathrm{f}$ occupied band in $\mathrm{NdScSiC}_{0.5}$ arises from both $\mathrm{Nd}-\mathrm{Sc}$ and $\mathrm{Nd}-\mathrm{C}$ chemical bonding. Therefore, carbon interacts significantly with $\mathrm{Nd}$ ( $\mathrm{f}$ and $\mathrm{d}$ orbitals) whereas $\mathrm{H} 2$ does not (Figure 16 (e-f)) despite being on the same 
site. Instead, $\mathrm{H} 2$ interacts strongly with $\mathrm{Sc}$, reducing significantly the $\mathrm{Nd}-\mathrm{Sc}$ coupling by competing chemical bonding.
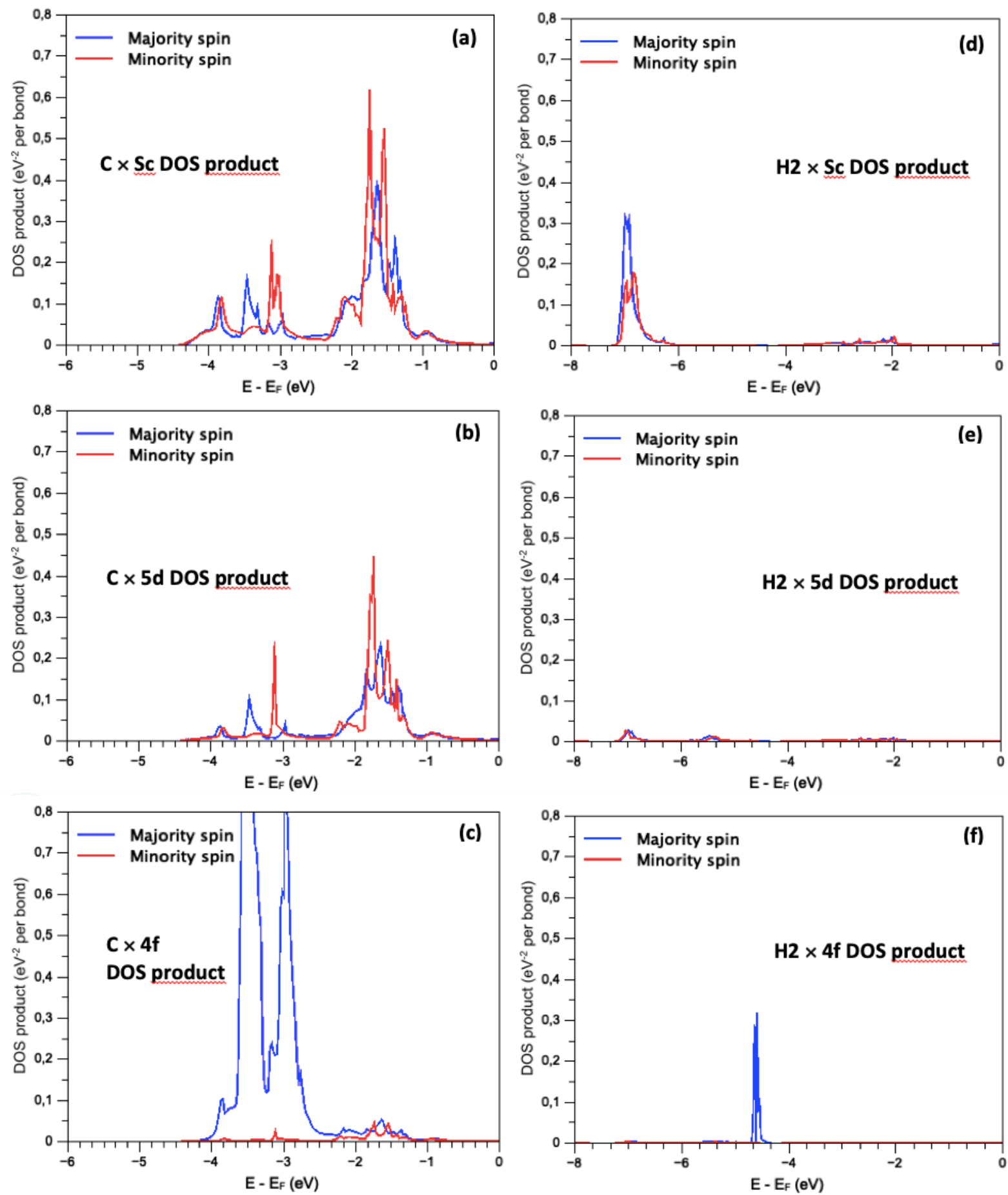

Figure 16. DOS products vs. energy (with respect to Fermi level), calculated from DFT/HSE06. (a),

(b) and (c): respectively C-Sc, C-5d(Nd) and C-4f(Nd) products in $\mathrm{NdScSiC}_{0.5 \cdot}(\mathrm{d})$, (e) and (f): respectively $\mathrm{H2}-\mathrm{Sc}, \mathrm{H2}-5 \mathrm{~d}(\mathrm{Nd})$ and $\mathrm{H2}-4 \mathrm{f}(\mathrm{Nd})$ products in $\mathrm{NdScSiH}_{1.5} . \mathrm{H} 2$ refers to the hydrogen atom located in the $\mathrm{Sc}_{4} \mathrm{Nd}_{2}$ octahedral site. For sake of comparison, the same scale for DOS products was used throughout the figure. 
These features are fully consistent with the occurrence of magnetic ordering ( $\mathrm{Nd}$ layers along the $c$-axis) at high temperature in $\mathrm{NdScSi}$ and $\mathrm{NdScSiC}_{0.5}$, and its disappearance in $\mathrm{NdScSiH}_{1.5}$. $\mathrm{Sc}$ in $\mathrm{NdScSi}$, and both $\mathrm{Sc}$ and $\mathrm{C}$ in $\mathrm{NdScSiC} \mathrm{C}_{0.5}$, mediate the magnetic interactions between $\mathrm{Nd}$ double layers, although with a quite different scenario in the two compounds. In the hydride, $\mathrm{H} 2-\mathrm{Sc}$ chemical bonding (and likely $\mathrm{Nd}-\mathrm{H} 1$, through competing interactions) ${ }^{6}$ kills the superexchange coupling of $\mathrm{Nd}$ double layers.

A way to investigate the spin contribution to the magnetic moment in these compounds is to plot the unpaired spin density (i.e. the difference between the electron density of majority and minority spin channels) in the unit cell, for a selected isovalue. Figure 17 shows that the differences in spin densities are striking, with continuous $\mathrm{Nd}$-Sc d-character paths in $\mathrm{NdScSi}$, which disappear in $\mathrm{NdScSiC}_{0.5}$ and $\mathrm{NdScSiH}_{1.5}$. This drastic effect of hydrogen was also observed in GdScGe vs. GdScGeH. ${ }^{6}$
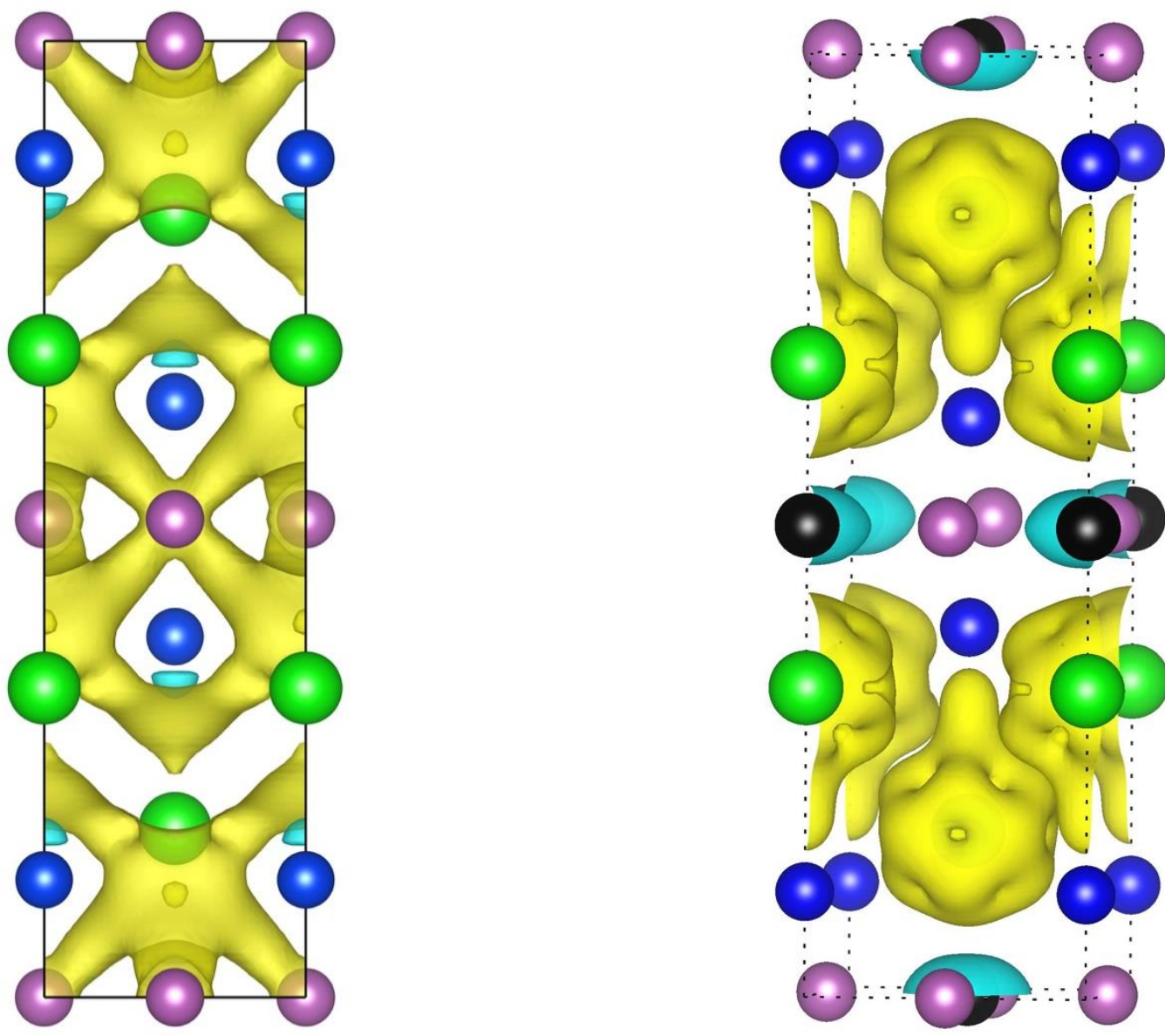

Figure 17. Isovalue plots of the unpaired spin density for $\mathrm{NdScSi}$ (left), $\mathrm{NdScSiH}{ }_{1.5}$ (middle) and $\mathrm{NdScSiC}_{0.5}$ (right), calculated from DFT/HSE06. Yellow and blue surfaces correspond respectively to positive and negative values of the spin density (same absolute value). Green, purple, blue, red and black spheres represent $\mathrm{Nd}, \mathrm{Sc}, \mathrm{Si}, \mathrm{H}$ and $\mathrm{C}$ atoms, respectively. Plots were made using the software VESTA. ${ }^{44}$ 
In $\mathrm{NdScSiC}_{0.5}$, antiferromagnetic $\mathrm{Nd}-\mathrm{C}$ coupling takes over vanished $\mathrm{Nd}-\mathrm{Sc}$ ferromagnetic interactions in $\mathrm{NdScSi}$. NdScSiH 1.5 shows reduced spin densities on $\mathrm{Nd}$, together with $\mathrm{Sc}-\mathrm{Sc}$ spin density paths within $\mathrm{ScH}_{0.5}$ layers likely decoupled from $\mathrm{NdH}$ layers (or weakly antiferromagnetically coupled). These distributions of spin density are consistent with DOS features described above: in particular, they correspond to the strong spin polarization of the DOS for NdScSi compared to $\mathrm{NdScSiC}_{0.5}$, where the spin polarization of Sc-character bands almost vanishes. Also, this indicates that Sc contributes to the ferromagnetic ordering in $\mathrm{NdScSi}$ by both coupling $\mathrm{Nd}$ double layers and carrying a significant magnetic moment. In the carbide, Sc contributes to couple Nd layers, but has no net spin population. Carbon has one, of opposite sign to neodymium.

\section{Conclusions}

$\mathrm{NdScSi}$ contains vacant interstitial sites that may be filled with carbon atoms. Single crystal diffraction indicates that the carbon atoms occupy the vacant $\mathrm{Sc}_{4} \mathrm{Nd}_{2}$ octahedral $2 b$ sites, leaving the $\mathrm{Nd}_{4}$ tetrahedral sites unoccupied. Carbon insertion causes an anisotropic expansion of the unit cell with the $a$ parameter increasing while the $c$ parameter decreases. The resulting $\mathrm{NdScSiC}_{x}$ carbide exists in a solid solution with $0.2<\mathrm{x} \leq 0.5$. Carbon insertion causes a linear decrease in the $T_{c}$ of the pristine compound from $171(2) \mathrm{K}$ (for $\mathrm{x}=0$ ) to 51 (3) $\mathrm{K}$ (for $\mathrm{x}=0.5$ ). The magnetic structures of $\mathrm{NdScSi}$ and $\mathrm{NdScSiC}_{0.5}$ were determined from neutron diffraction methods. Both $\mathrm{NdSCSi}$ and $\mathrm{NdScSiC}_{0.47(1)}$ demonstrate ferromagnetic magnetic structures. In $\mathrm{NdScSi}$ the $\mathrm{Nd}$ moments are aligned in the $a b$-plane while the $\mathrm{Nd}$ moments are aligned along the $c$-axis in the carbide.

DFT calculations suggest that a strong chemical bonding between $\mathrm{C}$ and $\mathrm{Nd}$ atoms exists in $\mathrm{NdScSiC}_{0.5}$ while the $\mathrm{H} 2-\mathrm{Nd}$ interaction is rather weak in $\mathrm{NdScSiH}_{1.5}, \mathrm{C}$ and $\mathrm{H} 2$ occupying equivalent position. The strong $\mathrm{C}-\mathrm{Nd}$ interaction results in a shortening of the $c$ lattice parameter through carbon insertion, enhancing the 3D character of the structure. This effect on $\mathrm{c}$ is not observed upon $\mathrm{H}$ insertion in $\mathrm{NdScSi}$, in agreement with weak $\mathrm{H} 2-\mathrm{Nd}$ chemical bonding, because $\mathrm{H} 1$ atoms in the the $\mathrm{Nd}_{4}$ tetrahedra induce an opposite trend with an increase of the $c$ parameter. Besides, the spectacular spin density path between $\mathrm{Nd}$ and Sc atoms in $\mathrm{NdScSi}$, which gives it a high Curie temperature, vanishes in the hydride and is significantly reduced in the carbide. Therefore, the magnetic ordering diseapppears in $\mathrm{NdScSiH}_{1.5}$; in $\mathrm{NdScSiC}_{0.5}$ the strong $\mathrm{Nd}-\mathrm{C}$ coupling connected to a smaller $c$ parameter, together with $\mathrm{Nd}$-Sc interactions of different nature (non spin-polarized) maintains magnetic interactions between $\mathrm{Nd}$ double layer and a ferromagnetic order at $50 \mathrm{~K}$ is still observed. The contribution of Sc moments to the magnetization vanishes in the carbide. Spin density maps reveal as well that the $\mathrm{Nd}-\mathrm{Sc}$ coupling is ferromagnetic in $\mathrm{NdScSi}$, while the $\mathrm{Nd}-\mathrm{C}$ coupling is antiferromagnetic in $\mathrm{NdScSiC} 0.5$. 
Supporting Information Available: Rietveld refinement of X-ray diffraction data, extra magnetization vs temperature curves.

\section{References}

(1) Gupta, S.; Suresh, K. G. Review on Magnetic and Related Properties of RTX Compounds. J. Alloys Compd. 2015, 618, 562-606.

https://doi.org/10.1016/j.jallcom.2014.08.079.

(2) Bernardini, F.; Garbarino, G.; Sulpice, A.; Núñez-Regueiro, M.; Gaudin, E.; Chevalier, B.; Méasson, M. A.; Cano, A.; Tencé, S. Iron-Based Superconductivity Extended to the Novel Silicide LaFeSiH. Phys. Rev. B 2018, 97 (10), 2-6.

https://doi.org/10.1103/PhysRevB.97.100504.

(3) Gaudin, E.; Matar, S. F.; Pöttgen, R.; Eul, M.; Chevalier, B. Drastic Change of the Ferromagnetic Properties of the Ternary Germanide GdTiGe through Hydrogen Insertion. Inorg. Chem. 2011, 50 (21), 11046-11054. https://doi.org/10.1021/ic201579r.

(4) Tskhadadze, I. A.; Chernyshev, V. V.; Streletskii, A. N.; Portnoy, V. K.; Leonov, A. V.; Sviridov, I. A.; Telegina, I. V.; Verbetskii, V. N.; Seropegin, Y. D. D.; Morozkin, A. V. GdTiGe (CeScSi-TYPE STRUCTURE) AND GdTiGe (CeFeSi-TYPE STRUCTURE) AS THE COHERENT PHASES WITH DIFFERENT MAGNETIC AND HYDROGENIZATION PROPERTIES. Mater. Res. Bull. 1999, 34 (99), 1773-1787.

(5) Chevalier, B.; Hermes, W.; Heying, B.; Rodewald, U. C.; Hammerschmidt, A.; Matar, S. F.; Gaudin, E.; Pöttgen, R. New Hydrides REScSiH and REScGeH (RE = La, Ce): Structure, Magnetism, and Chemical Bonding. Chem. Mater. 2010, 22 (17), 50135021. https://doi.org/10.1021/cm101290f.

(6) Mahon, T.; Gaudin, E.; Villesuzanne, A.; Decourt, R.; Bobet, J. L.; Isnard, O.; Chevalier, B.; Tencé, S. Hydrogen Insertion in the Intermetallic GdScGe: A Drastic Reduction of the Dimensionality of the Magnetic and Transport Properties. Inorg. Chem. 2018, 57 (22), 14230-14239. https://doi.org/10.1021/acs.inorgchem.8b02247.

(7) Tencé, S.; Mahon, T.; Gaudin, E.; Chevalier, B.; Bobet, J.-L. J.-L.; Flacau, R.; Heying, B.; Rodewald, U. C.; Pöttgen, R. Hydrogenation Studies on NdScSi and NdScGe. J. Solid State Chem. 2016, 242, 168-174. https://doi.org/10.1016/j.jssc.2016.02.017.

(8) Mahon, T.; Tencé, S.; Pöttgen, R.; Chevalier, B.; Gaudin, E. Study of the Structural Transition and Hydrogenation of CeTiGe. J. Alloys Compd. 2019, 805, 701-708. https://doi.org/10.1016/j.jallcom.2019.07.104.

(9) Wu, J.; Gong, Y.; Inoshita, T.; Fredrickson, D. C.; Wang, J.; Lu, Y.; Kitano, M.; Hosono, $\mathrm{H}$. Tiered Electron Anions in Multiple Voids of LaScSi and Their Applications to Ammonia Synthesis. Adv. Mater. 2017, 29 (36), 1700924.

https://doi.org/10.1002/adma.201700924. 
(10) Stassen, W. N.; Sato, M.; Calvert, L. D. The Crystal Structure of La2Sb. Acta Crystallogr Sect B 1970, 26 (10), 1534-1540. https://doi.org/10.1107/S0567740870004442.

(11) Mizoguchi, H.; Park, S. W.; Hiraka, H.; Ikeda, K.; Otomo, T.; Hosono, H. An Anti CuO2Type Metal Hydride Square Net Structure in Ln2M2As2 Hx ( $\mathrm{Ln}=\mathrm{La}$ or Sm, M = Ti, V, Cr, or Mn). Angew. Chemie - Int. Ed. 2015, 54 (10), 2932-2935.

https://doi.org/10.1002/anie.201409023.

(12) Mayer, I.; Shidlovsky, I. M5X3-Type Rare Earth Silicides and Germanides and Their Ternary Phases with Carbon. Inorg. Chem. 1969, 8 (6), 1240-1243.

https://doi.org/10.1021/ic50076a008.

(13) Ritter, C.; Wrubl, F.; Hill, A. H.; Pani, M.; Manfrinetti, P. Crystal and Magnetic Structure of the R15Si9C Compounds ( $\mathrm{R}=\mathrm{Ho}, \mathrm{Er}, \mathrm{Tb})$. J. Phys. Condens. Matter 2011, 23 (29). https://doi.org/10.1088/0953-8984/23/29/296002.

(14) Tencé, S.; Isnard, O.; Wrubl, F.; Manfrinetti, P. A Neutron Diffraction Study of the R15Ge9C Compounds ( $\mathrm{R}=\mathrm{Ce}$, $\mathrm{Pr}$, Nd). J. Alloys Compd. 2014, 594, 148-152. https://doi.org/10.1016/j.jallcom.2014.01.115.

(15) Shcherbakova, Y. V.; Ivanova, G. V.; Gaviko, V. S.; Gabay, A. M. Crystal Structure of Novel Ferromagnetic LaFe ${ }_{13-x} G_{x}$ C Compounds. J. Magn. Magn. Mater. 2003, 267, 26-34. https://doi.org/10.1016/S0304-8853(03)00300-7.

(16) Zhang, H.; Hu, F. X.; Sun, J. R.; Shen, B. G. Effects of Interstitial H and/or C Atoms on the Magnetic and Magnetocaloric Properties of La(Fe, Si)13-Based Compounds. Science China: Physics, Mechanics and Astronomy. Springer Berlin Heidelberg December 2013, pp 2302-2311. https://doi.org/10.1007/s11433-013-5357-1.

(17) Coey, J. M. D.; Hurley, D. P. F. New Interstitial Rare-Earth Iron Intermetallics Produced by Gas Phase Reaction. J. Magn. Magn. Mater. 1992, 104-107 (PART 2), 1098-1101. https://doi.org/10.1016/0304-8853(92)90506-J.

(18) Fruchart, D.; Isnard, O.; Miraglia, S.; Pontonnier, L.; Soubeyroux, J. L.; Fruchart, R. $\mathrm{R}_{2} \mathrm{Fe}_{17}$ and $\mathrm{RM}_{12}$ Carbide and Carbonitride Synthesis from Heavy Hydrocarbon Compounds. J. Alloys Compd. 1994, 203 (C), 157-163. https://doi.org/10.1016/09258388(94)90728-5.

(19) De Mooij, D. B.; Buschow, K. H. J. Formation and Magnetic Properties of the Compounds R2Fe14C. J. Less Common Met. 1988, 142, 349-357. https://doi.org/10.1016/0022-5088(88)90194-4.

(20) Coey, J. M. D. Novel Permanent Magnetic Materials. Phys. Scr. 1991, T39, 21-28. https://doi.org/10.1088/0031-8949/1991/T39/002.

(21) Khazzan, S.; Mliki, N.; Bessais, L.; Djéga-Mariadassou, C. Rare-Earth Iron-Based Intermetallic Compounds and Their Carbides: Structure and Magnetic Behaviors. J. Magn. Magn. Mater. 2010, 322 (2), 224-229. https://doi.org/10.1016/j.jmmm.2009.08.047.

(22) Klosek, V. Thesis Université Henri Poincaré-Nancy I, 2002. 
(23) Lemoine, P. Thesis Université Henri Poincaré-Nancy I, 2011.

(24) Tencé, S.; Gaudin, E.; Isnard, O.; Chevalier, B. Magnetic and Magnetocaloric Properties of the High-Temperature Modification of TbTiGe. J. Phys. Condens. Matter 2012, 24 (29). https://doi.org/10.1088/0953-8984/24/29/296002.

(25) Rodriguez-Carvajal, J. Recent Advances in Magnetic Structure Determination by Neturon Powder Diffraction. Phys. B 1993, 192, 55-69.

(26) Petrícek, V.; Dušek, M.; Palatinus, L. Crystallographic Computing System JANA2006: General Features. Zeitschrift fur Krist. 2014, 229 (5), 345-352. https://doi.org/10.1515/zkri-2014-1737.

(27) Kresse, G.; Hafner, J. Ab Initio Molecular Dynamcis for Liquid Metals. Phys. Rev. B 1993, 47 (1), 558.

(28) Furthmüller, J.; Kresse, G. Efficiency of Ab-Initio Total Energy Calculations for Metals and Semiconductors Using a Plane-Wave Basis Set. Comput. Mater. Sci. 1996, 6 (1), 15-50. https://doi.org/10.1016/0927-0256(96)00008-0.

(29) Blochl, P. E. Projector Augmented-Wave Method. Phys. Rev. B 1994, 50 (24).

(30) Perdew, J. P.; Ernzerhof, M.; Burke, K. [ERRATA] Generalized Gradient Approximation Made Simple. Phys. Rev. Lett. 1997, 78 (18), 3865-3868.

https://doi.org/10.1103/PhysRevLett.77.3865.

(31) Heyd, J.; Scuseria, G. E.; Ernzerhof, M. Hybrid Functionals Based on a Screened Coulomb Potential. J. Chem. Phys. 2003, 118 (18), 8207-8215. https://doi.org/10.1063/1.1564060.

(32) Krukau, A. V.; Vydrov, O. A.; Izmaylov, A. F.; Scuseria, G. E. Influence of the Exchange Screening Parameter on the Performance of Screened Hybrid Functionals. J. Chem. Phys. 2006, 125 (22), 0-5. https://doi.org/10.1063/1.2404663.

(33) Paier, J.; Marsman, M.; Hummer, K.; Kresse, G.; Gerber, I. C.; Angyán, J. G. Screened Hybrid Density Functionals Applied to Solids. J. Chem. Phys. 2006, 124 (15). https://doi.org/10.1063/1.2187006.

(34) Manfrinetti, P.; Pani, M.; Palenzona, A.; Dhar, S. K.; Singh, S. Single Crystal Study of the High-Curie-Temperature Ferromagnet Gd Sc Ge and of Gd Sc Ge 1.020 .982 .38 2.62 3. J. Alloys Compd. 2002, 334, 9-13.

(35) Atoji, M. Crystals and Magnetic Structures of $\mathrm{CeC}_{2}, \mathrm{PrC}_{2}$ and $\mathrm{NdC}_{2}$. Phys. Lett. 1966, 22 (1), 21-22. https://doi.org/10.1016/0031-9163(66)90039-4.

(36) Atoji, M. Neutron Diffraction Studies of $\operatorname{Pr}_{2} \mathrm{C}_{3}, \mathrm{Nd}_{2} \mathrm{C}_{3}$, and $\mathrm{Dy}_{2} \mathrm{C}_{3}$ at 300 to 1.6K. J. Solid State Chem. 1978, 26 (1), 51-57. https://doi.org/10.1016/0022-4596(78)90132-9.

(37) Nowotny, H.; Auer-Welsbach, H. Uber Das Scandiumcarbid. Monatshefte fur Chemie 1961, 92 (3), 789-793. https://doi.org/10.1007/BF00918639.

(38) Pöttgen, R.; Jeitschko, W. Scandium Carbide, $\mathrm{Sc}_{3} \mathrm{C}_{4}$, a Carbide with $\mathrm{C}_{3}$ Units Derived 
from Propadiene. Inorg. Chem. 1991, 30 (3), 427-431.

https://doi.org/10.1021/ic00003a013.

(39) Krikorian, N. H.; Bowman, A. L.; Krupka, M. C.; Arnold, G. P. The Preparation and Crystal Structure of $\mathrm{Sc}_{4} \mathrm{C}_{3}$. High Temp. Sci. 1969, 1, 360-366.

(40) Jedlicka, H.; Nowotny, H.; Benesovsky, F. Zum System Scandium-Kohlenstoff, 2. Mitt.: Kristallstruktur Des C-Reichen Carbids. Monatshefte fur Chemie 1971, 102 (2), 389403. https://doi.org/10.1007/BF00909332.

(41) Singh, S.; Dhar, S. K.; Manfrinetti, P.; Palenzona, A.; Mazzone, D. High Magnetic Transition Temperatures in RScT ( $\mathrm{R}=\mathrm{Pr}$, Nd and Sm; T = Si and Ge) Compounds: Multiple Spin Reorientations in PrScGe. J. Magn. Magn. Mater. 2004, 269 (1), 113121. https://doi.org/10.1016/S0304-8853(03)00583-3.

(42) Boulet, P.; Weitzer, F.; Hiebl, K.; Noel, H. Structural Chemistry, Magnetism and Electrical Properties of Binary Nd Silicides. J. Alloys Compd. 2001, 315 (1-2), 75-81. https://doi.org/10.1016/S0925-8388(00)01304-9.

(43) Manfrinetti, P.; Morozkin, A. V.; Isnard, O.; Henry, P.; Palenzona, A. Magnetic Structure of the CeScSi-Type RScGe Compounds ( $\mathrm{R}=\mathrm{Pr}$, Nd, Tb). J. Alloys Compd. 2008, 450 (1-2), 86-91. https://doi.org/10.1016/j.jallcom.2006.11.057.

(44) Momma, K.; Izumi, F. VESTA 3 for Three-Dimensional Visualization of Crystal, Volumetric and Morphology Data. J. Appl. Crystallogr. 2011, 44 (6), 1272-1276. https://doi.org/10.1107/S0021889811038970.

\section{For Table of Content Only}
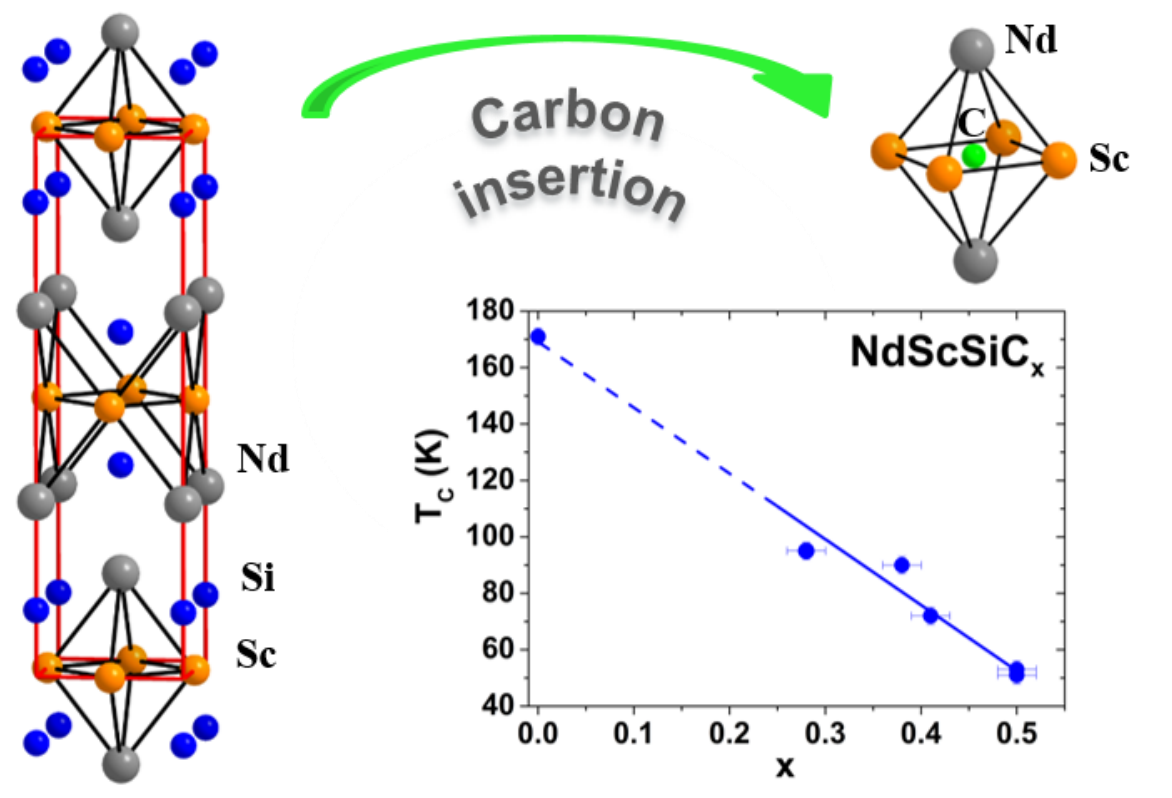


\section{Synopsis}

We present the first carbide compound, $\mathrm{NdScSiC} 0.5$, obtained by carbon insertion in an equiatomic intermetallic $R T X$ ( $R=$ rare-earth, $T=$ transition metal, $X=\mathrm{p}$ element). Carbon atoms occupy the vacant $\mathrm{Sc}_{4} \mathrm{Nd}_{2}$ octahedral sites of $\mathrm{NdScSi}$. The strong $\mathrm{Nd}-\mathrm{C}$ chemical bonding enhances the 3D character of the structure and maintains a ferromagnetic order although the $\mathrm{Nd}-\mathrm{Sc}$ interactions are no more spin-polarized compared to those in $\mathrm{NdScSi}$. 